\title{
GM-CSF signaling is critical for HER2+ breast leptomeningeal carcinomatosis
}

\section{Khairul Ansari}

Celcuity https://orcid.org/0000-0002-9564-2107

Arunoday Bhan

City Of Hope National Medical Center

Mike Chen

City of Hope

Rahul Jandial ( $\sim$ Rjandial@coh.org)

City Of Hope National Medical Center

\section{Article}

Keywords: Leptomeningeal carcinomatosis, HER2+ breast cancer, HER2+ LC

Posted Date: September 9th, 2020

DOI: https://doi.org/10.21203/rs.3.rs-65694/v1

License: @ (i) This work is licensed under a Creative Commons Attribution 4.0 International License. Read Full License 
1 GM-CSF signaling is critical for HER2+ breast leptomeningeal carcinomatosis

Authors: Khairul Ansari1,2*, Arunoday Bhan1*, Mike Y. Chen1, Rahul Jandial1

\section{Affiliations:}

6 1Division of Neurosurgery, Beckman Research Institute, City of Hope, Duarte, CA 91010, USA

$7 \quad{ }_{2}$ Celcuity LLC, Minneapolis, MN 55446, USA

8 *Equally contributing co-authors

9

10 Corresponding author: Rahul Jandial; Rjandial@coh.org 
Leptomeningeal carcinomatosis (LC), when tumor cells spread to leptomeninges surrounding the brain 4 and spinal cord. HER2+ breast cancer is the most common origin of LC. HER2+ LC remains incurable, with few 5 treatment options and response rates of $<20 \%$. One major limitation in development of HER $2+$ LC therapies has 6 been lack of clinically relevant HER $2+$ LC primary cell-lines and animal models. To address this, we generated 7 cell lines and patient-derived xenograft models using nodular HER2+ LC. This led to identification of 8 granulocyte-macrophage colony-stimulating factor (GM-CSF) as an oncogenic autocrine driver of HER2+ LC. 9 We observed that oligodendrocyte progenitor cells (OPCs) inhibit growth of HER2+ LC in vitro and in vivo. 10 Furthermore, OPC-derived factor TPP1 degrades GM-CSF, decreasing GM-CSF signaling and suppressing 11 HER2+ LC growth. Lastly, we determined that synergistic inactivation of GM-CSF signaling via the intrathecal 12 delivery neutralizing anti-GM-CSF antibodies and a pan-Aurora kinase inhibitor (CCT137690) antagonizes 13 development of HER2+ LC in vivo. 


\section{$1 \quad$ KEY POINTS}

2 A. We derived the first-ever HER2+ breast cancer leptomeningeal carcinomatosis (HER2+ LC) cell lines and 3 patient-derived xenograft mouse models.

4 B. Granulocyte-macrophage colony-stimulating factor (GM-CSF) is an oncogenic autocrine driver of HER2+ $5 \quad$ LC.

6 C. The oligodendrocyte progenitor cell-derived factor TPP1 degrades GM-CSF, decreasing GM-CSF signaling 7 and suppressing HER2+ LC growth.

8 D. Synergistic inactivation of GM-CSF signaling using neutralizing anti-GM-CSF antibodies and a pan-Aurora 9 kinase inhibitor (CCT137690) antagonizes the development of HER2+ LC. 


\section{Introduction}

HER2+ breast cancer leptomeningeal carcinomatosis (HER2+ LC) is an ominous complication of breast cancer with a dire prognosis $1,2,3.10-30 \%$ of patients with metastatic breast tumors develop CNS metastases4, 5. HER2+ LC occurs when HER2+ breast tumor cells spread to the cerebrospinal fluid (CSF)-containing leptomeninges surrounding the brain and spinal cord $1,2,3,6$. Several factors positively correlate with a greater risk of brain metastases, including young age; poorly differentiated tumors; HER2-enriched, luminal-HER2, basallike, and TN breast cancer subtypes; and having four or more metastatic lymph-nodes7, 8. Once established, HER2+ LC can invade the parenchyma to produce focal neurologic damage9. Any malignancy can spread to the leptomeninges; however, given the high incidence of breast cancer (and particularly the HER2+ subtype) worldwide, breast cancer is the most common origin of $\mathrm{LC}_{10}$. Although significant progress has been made in developing breast cancer treatments that target systemic disease, efficacy in the central nervous system (CNS) remains a challenge, with HER2+ LC typically developing while the systemic tumor burden is well-managed11, 12, 13, 14. Indeed, $30 \%$ of HER2+ LC cases are diagnosed as the first manifestation of cancer after a substantial disease-free interval13, 15, 16, 17.

Unfortunately, although it was discovered nearly 150 years ago18, HER2+ LC remains incurable, with few treatment options and response rates often $<20 \% 19,20,21,22,23,24,25$. At present, HER2+ LC management is multidisciplinary and includes radiotherapy (RT) and intrathecal chemotherapy (ITC) 26, 27, 28, 29. Methotrexate (MTX), a DNA alkylating drug, is frequently used as palliative ITC for HER2+ LC9, 30, 31, 32, 33. However, this approach has very limited success and causes serious side effects26, 27, 28, 29. Furthermore, patients with HER2+ LC are excluded from clinical trials because of their poor prognosis and to minimize results that are not reproducible1, 34,35,36. Thus, our capacity to identify effective drugs to treat HER2+ LC is limited.

In addition, little is known about how HER2+ breast cancer cells proliferate in the compositionally simple context of the leptomeninges, which are acellular and poor in protein, glucose, and cytokine content1, 2, 3,6,. This lack of understanding regarding the molecular mechanisms that govern HER2+ LC development is due in part to a lack of clinically relevant primary HER2+ LC cell lines and patient-derived xenograft (PDX) models. Until now, HER2+ LC analysis has been conducted primarily using floating HER2+ LC cells removed from the CSF 
1 of patients in numbers insufficient to generate cell lines. Moreover, these floating cells differ from nodular tissue, 2 which represents the major clinical burden of HER2+ LC.

3 To address this limitation, we generated the first-ever expandable primary HER2+ LC cell lines and PDX 4 models. Using these models, we observed a non-uniform distribution of HER2+ LC inside the brain and spinal 5 cord, with a paucity of HER2+ LC metastases in white matter compared to the astrocyte-rich gray matter of the 6 cerebral hemispheres. This pattern recapitulates the distribution of metastases in the human CNS, where the 7 preponderance of metastases is in gray matter regions, with scarcely any in white matter. Further analysis revealed 8 that granulocyte-macrophage colony-stimulating factor (GM-CSF) is an oncogenic autocrine factor that promotes 9 HER 2+ LC development in the CSF. In addition, we observed that oligodendrocyte precursor cells (OPCs), which 10 are abundant in white matter, disrupt GM-CSF HER2+ LC-derived oncogenic autocrine signaling, likely by 11 promoting GM-CSF degradation via TriPeptide Peptidase 1 (TPP1). Finally, we found that the synergistic 12 inactivation of GM-CSF signaling using anti-GM-CSF neutralizing antibodies and a pan-Aurora kinase inhibitor 13 (CCT137690) abrogates HER2+ LC growth both in vitro (using our novel HER2+ LC lines) and in vivo (using 14 our HER2+ LC PDX models). These findings bring us closer to discovering and developing more effective, novel 15 approaches for treating patients with HER2+ LC in the clinic. 


\section{Results}

\section{Derivation and characterization of nodular HER2+ LC cells}

HER2+ LC tumors, we dissociated fresh HER2+ nodular LC tissues obtained surgically for pathological confirmation of LC or to decompress localized symptomatic lesions. The tissues were dissociated into single cells by mechanical and enzymatic methods and then expanded on collagen-coated plates in media supplemented with patients and scaled them up to produce a cryobank of low-passage cells used for the experiments described herein. Based on our observations that Lepto1 and Lepto2 cells behave similarly both in vitro and in vivo, we have presented data primarily from the Lepto1 line.

To compare the metastatic organotropism of the primary Lepto lines, we first transduced them, as well as the brain-tropic breast cancer cell line MDA-MB-231 (231-BR cells), with lentiviruses encoding mCherry and firefly luciferase (mCherry:LUC). We then injected the cells (100K from each line) into the CSF space via the cisterna magna puncture of NOD/SCID mice $(n=12)$ and monitored tumor cell growth over time $(\sim 50$ days $)$ by BLI. As expected, 231-BR cells generated tumors only within the brain parenchyma37, 38. In contrast, Lepto1 bioluminescence appeared on the surface of the brain and spinal cord (Fig. 1B). In addition, although we observed fewer metastatic Leptol cells than 231-BR cells in the brain, there were overall more metastatic Lepto1 cells across both the brain and spinal cord (Fig. 1B). Furthermore, Kaplan-Meier curve analysis showed that mice harboring Lepto1 cells had significantly shorter survival periods than mice bearing 231-BR cells (Fig. 1C). Importantly, the transcriptomic analysis demonstrated that Lepto1 lines retrieved post-implantation from the leptomeninges of NOD/SCID mice exhibited gene signatures similar to those of pre-implanted primary Lepto1 cells (Fig. 1D).

Histological examination confirmed that Lepto1 cells colonized the leptomeningeal surface of the brain, brain stem, and spinal cord (Fig. 1E). Moreover, we observed no Lepto1 cells were observed inside of the white matter-rich brain stem and spinal cord (Fig. 1E). To confirm their regional preference (white vs. gray matter), we implanted Lepto1 cells into the brain stem (white matter) or cerebral cortex (gray matter) of NOD/SCID mice. 
1 BLI showed larger metastatic tumor deposits in mice with Lepto1 cells implanted in the cerebral cortex than in

2 mice with Lepto1 cells implanted in the brain stem (Fig. 1F). Furthermore, Lepto1 cell implantation into the

3 cerebral cortex significantly shortened animal survival compared to implantation into the brain stem (Fig. 1F).

4 Consistent with findings in patients that white matter is relatively tumor-resistant39 (Fig. 1G), we observed Lepto1

5 cell grafting efficiency of only $\sim 5 \%$ (1/16) after implantation into white matter, compared to 95\% (15/16) after

6 implantation into gray matter (Fig. 1H). In addition, the immunofluorescence-based analysis of mCherry: LUC-

7 labeled Lepto1 cells and their neighboring glial cell populations demonstrated that the Lepto1 cells were

8 juxtaposed to reactive (GFAP+) astrocytes in the brain (Suppl. Fig. 1A). A similar analysis revealed that Lepto1

9 cells were similarly juxtaposed to a layer of reactive (GFAP+) astrocytes on the surface of the brain stem and

10 spinal cord (Fig. 1I). In contrast, we observed that the inner core of the tissue, which is rich in OPCs, was devoid

11 of mCherry+ Lepto1 cells (Fig. 1H), suggesting that white matter resists Lepto1 cells colonization.

\section{OPCs reduce HER2+ LC cell viability}

To determine whether host glial cells modulate the heterogeneous distribution of metastases in the human

CNS, we immuno-panned CNS cell types from human iPSC-derived NPCs. Specifically, we used anti-CD45, anti-GALC, anti-CD90, anti-NG2, and anti-HepaCAM antibodies to FACS sort microglia, oligodendrocytes, neurons, OPCs, and astrocytes, respectively (Fig. 2A)40. Cells were maintained in hCSF for various durations, during which all cell types maintained their typical morphology and marker expression patterns (Fig. 2A). We then co-cultured the Lepto1 or Lepto2 cells along with each glial cell type in Boyden chambers to assess the influence of the various cell populations on their viability (Fig. 2B). Co-culture of both Lepto1 and Lepto2 cells with astrocytes increased their rate of proliferation, whereas co-culture with OPCs reduced their proliferation and induced apoptosis (Fig. 2B, 2C, and Suppl. Fig. 1D). Because Lepto lines can initiate and drive tumorigenesis (Fig. 1B, 1F), we performed tumorsphere formation assays to assess the effects of astrocytes or OPCs on the tumor-initiating capacity of Lepto1 cells. We co-cultured Lepto1 cells with astrocytes or OPCs for $72 \mathrm{~h}$ and then allowed the Lepto1 cells to generate spheroids on adherent culture plates. After 7 days, control (mono-cultured) 
1 greater quantity and size. In contrast, Lepto1 cells co-cultured with OPCs either remained as single cells or formed

2 very small spheroids (Suppl. Fig. 1B). Furthermore, quantitative analysis of cell viability using CCK assays 3 showed that, compared to mono-culture, co-culture with OPCs reduced the proportion of sphere-initiating Lepto1 4 cells to about 10\%, whereas co-culture with astrocytes increased cell viability (Suppl. Fig. 1C). Co-culture with 5 Lepto1 cells did not alter the proliferation of microglia, oligodendrocytes, neurons, OPCs, or astrocytes (Fig. 2D).

To characterize the effects of OPCs on Lepto1 cells in vivo, we injected mCherry:LUC-labeled Lepto1 cells $(100 \mathrm{~K})$ into the cisternae magna of adult NOD/SCID mice (Day 0). We then injected OPCs (100K) into the cisternae magna on Days 7 and 14 (Fig. 2E) and monitored tumor growth via BLI from Days 14 to 50. Mice coimplanted with Lepto1 cells followed by OPCs showed reduced tumor growth, based on BLI, and prolonged survival relative to mice that received Lepto1 cells only (Fig. $\mathbf{2 F}$ and $\mathbf{2 G}$ ). This finding confirms the inhibitory properties of OPCs seen in vitro and strongly suggests that intrathecal OPC delivery suppresses HER2+ LC 12 progression in vivo.

\section{GM-CSF acts as an oncogenic autocrine driver of HER2+ LC cell growth}

To determine how Lepto cells initiate tumors and drive tumorigenesis in the leptomeningeal space surrounding the brain and spinal cord, we co-cultured Lepto1 cells with OPCs for $72 \mathrm{~h}$ in a Boyden chamber and compared them to Lepto1 control cells grown for the same duration as independent monolayers. When we subjected growth medium from each sample to immunoblotting using a Cytokine XL array, we observed significantly higher GM-CSF levels in the growth media of mono-cultured Lepto1 cells than in that of co-cultured OPC/Lepto1 cells (Fig. 3A, 3B). Indeed, GM-CSF is expressed at higher levels in Lepto1 cells than in any of the CNS cell types analyzed (Suppl. Fig. 2A) and was elevated in nodular HER2+ LC tissues compared to other primary tumor, normal breast, and normal brain tissues (Suppl. Fig. 2A). Furthermore, surface GM-CSF receptors (GM-CSFR $\alpha$, or CD116) were homogenously expressed on $~ 99 \%$ of Lepto1 cells (Suppl. Fig. 2B), suggesting autocrine activation of the GM-CSF signaling pathway in Lepto1 lines via binding of Lepto1-secreted GM-CSF. Furthermore, western blot analysis of cultured Lepto1 cells showed that the presence of OPCs suppressed the phosphorylation-dependent activation of GM-CSFR $\alpha$ and its downstream targets, STAT5, AKT, and ERK1/2 
1 (Fig. 3C). Furthermore, immunohistochemical analyses of pGM-CSFR $\alpha$ revealed receptor activation in tumor

2 tissue but not in surrounding brain tissue (Fig. 3D). Overall, these analyses suggest that OPCs inhibit the secretion

3 of GM-CSF from Lepto1 cells, which in turn inhibits the activation of GM-CSFR $\alpha$ and its effectors.

4 To test this hypothesis, we analyzed GM-CSF signaling in Lepto1 cells grown in either hCSF5 supplemented media (controls), OPC-conditioned hCSF, or hCSF containing anti-GM-CSF neutralizing 6 antibodies (Fig. 3E, 3F). Based on immunoblot analysis, GM-CSF signaling was disrupted in Lepto1 cells 7 cultured in OPC-conditioned media or in hCSF with GM-CSF neutralizing antibodies (Fig. 3E), and cells in both 8 conditions were significantly more apoptotic than control Lepto1 cells (Fig. 3F). Conditional overexpression of 9 GM-CSF in Lepto1 cells using a Tet-On 3G inducible expression system with ZsGreenI (Suppl. Fig. 2C-E) not 10 only decreased the OPC-mediated induction of apoptosis (Suppl. Fig 2F) but also reduced the OPC-induced 11 suppression of Lepto1 cell viability (Suppl. Fig. 2G). To determine whether OPC-derived or secreted factors inhibit GM-CSF expression and secretion by Lepto1 cells, we generated a luciferase reporter construct containing the GM-CSF proximal promoter (+1 to -500 bp) upstream of the luciferase gene. We then transfected Lepto1 and Lepto2 cells with this construct and co-cultured them with OPC cells, OPC-conditioned media, or control media (Suppl. Fig. 2I). Interestingly, luciferase activity (Suppl. Fig. 2I) and GM-CSF expression (Suppl. Fig. 2H) were comparable across all experimental and control groups, suggesting that OPCs or OPC-derived factors do not regulate GM-CSF expression. These results suggest that OPCs secrete factors that inhibit GM-CSF signaling via a different mechanism (Fig. 3G).

Anti-GM-CSF neutralizing antibodies have been used clinically in cancer treatment 41,42 . Therefore, we assessed the potential anti-tumor effects of anti-GM-CSF neutralizing antibodies in our HER2+ LC PDX model, administering the antibodies intrathecally and analyzing HER2+ LC formation by BLI (Fig. 3H). Relative to vehicle-treated controls, antibody-treated mice showed reduced tumor progression and increased overall survival (Fig. 3I). Moreover, immunohistochemical analysis of brain sections (Fig. 3J) and western blot analysis of extracted tumor lysates (Fig. 3K) revealed that intrathecal administration of anti-GM-CSF neutralizing antibodies suppressed not only canonical GM-CSFR $\alpha$ activation and EGFR phosphorylation but also the phosphorylationmediated activation of STAT5, AKT, and mTOR (downstream GM-CSF pathway effectors43, 44) in HER2+ LC 
1 PDX tumors. Taken together, these results confirm the critical role of GM-CSF signaling in HER2+ LC tumor

2 progression and suggest that treatment with anti-GM-CSF neutralizing antibodies could serve as a potential 3 strategy to target HER2+ LC.

5 OPC-derived TPP1 is a candidate regulator of GM-CSF in HER2+ LC cells

Given that OPCs suppress HER2+ LC cell viability and tumorsphere initiation in vitro (Fig. 2B, 2C) and inhibit HER2+ LC tumor growth in vivo (Fig. 2F), we asked whether OPCs secrete factors that inhibit GM-CSF signaling and induce Lepto cell apoptosis. To do so, we used liquid chromatography-tandem mass spectrometry (LC-MS/MS) to analyze the secretomes of human astrocytes or OPCs, cultured either alone or with Lepto1 cells.

We identified 38 unique proteins in OPC-conditioned hCSF secreted exclusively by OPCs and whose levels remained unchanged in OPC-Lepto1 co-cultures (Fig. 4A and Supplementary Table 1). Among them, only CGREF1, ENO, PTPRZ1, SPARC, and TPP1 proteins have been previosuly identified as secreted proteins (Supplementary Table 1). None of the identified proteins with extracellular localization can bind to or chelate GM-CSF, and only TPP1 (Fig. 4B), a serine protease in the sedolisin family45, possesses the ability to degrade GM-CSF. Therefore, we hypothesized that OPC-derived TPP1 could proteolytically degrade GM-CSF, leading to suppression of the GM-CSF signaling pathway and consequent inhibition of Lepto1 cell growth and viability. To determine whether TPP1 regulates GM-CSF signaling, we incubated Lepto1 cells with 50 or $100 \mathrm{ng} / \mathrm{mL}$ of recombinant TPP1 protein in hCSF for 24 hours and monitored GM-CSF levels by ELISA. Both concentrations of TPP1 significantly reduced GM-CSF levels in Lepto1 cells (Fig. 4C) and suppressed cell viability (Fig. 4D). TPP1 treatment (100 ng/mL) also reduced the phosphorylation and hence the activation of the GM-CSF effectors STAT5, AKT, and mTOR (Fig. 4E). To determine the effects of this suppression, we treated Lepto1 cells with an AKT inhibitor (AZD5363) or an mTOR inhibitor (Rapamycin), both currently in use or under development to target solid tumors46, 47, 48, 49, 50. Treatment with either suppressed Lepto1 cell viability with IC50 values in the nanomolar range (Suppl. Fig. 3A). Moreover, co-culture with OPCs and treatment with TPP1 (50 ng/mL) reduced GM-CSF levels in the culture media of Lepto1 cells conditionally overexpressing GM-CSF (following treatment with $5 \mu \mathrm{g} / \mathrm{mL}$ doxycycline, DOX), as measured by ELISA (Fig. 4F). Subsequent western blot analyses showed 
1 that co-culturing GM-CSF-overexpressing Lepto1 cells with OPCs and/or TPP1 (50 ng/mL), suppressed the

2 phosphorylation-dependent activation of GM-CSFR $\alpha$ and its downstream effectors, AKT and mTOR, suggesting

3 decreased oncogenic autocrine GM-CSF signaling (Fig. 4G). Futhermore, co-culturing Lepto1 cells with OPCs,

4 with and without TPP1 (50 ng/mL), induced apoptosis (Suppl. Fig. 3D), suggesting that OPC-derived TPP1

5 inhibits or degrades Lepto1-secreted GM-CSF and suppresses GM-CSF effectors.

To assess the effects of TPP1 loss-of-function on Lepto1 lines conditionally overexpressing GM-CSF, we transiently transduced OPC cells with siTPP1 and the following day co-cultured them (or control OPCs transduced with siGFP or siLUC) with the Lepto1 cells (Suppl. Fig. 3E). After one day of co-culture, siTPP1transduced OPC cells had significantly lower intracellular TPP1 levels than the control OPCs (Suppl. Fig. 3F). Interestingly, intracellular GM-CSF mRNA levels were not affected in Lepto1 cells or OPCs transduced with siTPP1, siGFP, or siLUC (Suppl. Fig. 3G). However, GM-CSF levels were significantly higher in media obtained from Lepto1 cells co-cultured with TPP1-depleted OPCs than in media from Lepto1 cells co-cultured with control

OPCs (Suppl. Fig. 3H). Similarly, the phosphorylation of signaling effectors downstream of GM-CSF was significantly greater in Lepto1 cells co-cultured with TPP1-depleted OPCs (Suppl. Fig. 3I).

To assess the effects of OPC-derived TPP1 on Lepto1 cells in vivo, we co-implanted Lepto1 cells and OPCs into the cisternae magna of NOD/SCID mice (Fig. 4H). As anticipated, we observed OPC densitydependent suppression of HER2+ LC tumor progression and augmented animal survival (Fig. 4I, J). Analyses of mouse CSF revealed that TPP1 protein levels also increased with increasing OPC density (Fig. 4K), accompanied by a corresponding decrease in GM-CSF levels (Fig. 4K). Interestingly, the OPCs co-implanted with Lepto1 cells did not exhibit altered viability between Days 5 and 20 and expressed appropriate cell-specific markers (Suppl. Fig. 3B and C). Overall, our in vitro (Fig. 4F) and in vivo (Fig. 4K) experiments suggest that OPC-secreted TPP1 degrades GM-CSF and suppresses GM-CSF signaling, thus decreasing Lepto1 cell viability and tumor progression.

Combination treatment with a pan-Aurora kinase inhibitor and anti-GM-CSF neutralizing antibodies antagonizes the growth of Lepto cells in vivo 
The Lepto lines derived from nodular HER2+ LC tissues mimic the metastatic organotropism of HER2+

2 LC seen in patients (Fig. 1). Thus, we investigated whether reagents selectively targeting the Lepto lines would 3 antagonize HER2+ LC in patients. To do so, we performed a chemical genetics screen using Lepto1 cells to assess 4 LOPAC-1280 compounds (Fig. 5A). Specifically, Lepto1 cells were seeded in 384-well plates at a density of 51,000 cells per well, and one day later, LOPAC-1280 compounds were added at 4 concentrations (100 nM, 200 $6 \mathrm{nM}, 400 \mathrm{nM}$, and 800nM in 0.1\% DMSO). After $72 \mathrm{~h}$, we analyzed cell viability using a CellTiter-Glo cell assay, 7 which indicated that both a pan-Aurora kinase inhibitor (CCT137690) and an SRC kinase inhibitor (Bosutinib) 8 inhibited Lepto1 cell viability by $\sim 95 \%$ (Fig. 5B). Dose-response assays showed that Lepto1 lines were sensitive

to CCT137690 and Bosutinib at all concentrations tested, but the IC50 value of CCT137690 was below $100 \mathrm{nM}$

(Fig. 5C), significantly lower than of Bosutinib. Thus, additional studies focused primarily on the effects of CCT137690. Treatment with CCT137690 (100 nM) significantly induced apoptosis in Lepto1 cells (Fig. 5D).

CCT137690 inhibits Aurora-A, B, and C kinases. Aurora-A participates in crosstalk between SRC kinase and GM-CSF signaling to regulate effectors such as STAT5, AKT, and mTOR, all of which play critical roles in Lepto1 cell proliferation and viability (Fig. 5E)51, 52, 53. Furthermore, we observed that Aurora-A levels were particularly elevated in nodular HER2+ LC tissues relative to other primary tumors or normal breast or brain tissues (Fig. 5F). In addition, western blot analysis of Lepto1 cells showed that combining anti-GM-CSF antibodies with either CCT137690 or Bosutinib suppressed the phosphorylation-dependent activation of GMCSF/GM-CSFR $\alpha$ effectors, including STAT5, AKT, and mTOR, thus reducing oncogenic autocrine GM-CSF signaling (Fig. 5G) and inducing Lepto1 cell apoptosis (Suppl. Fig. 4E).

Next, we investigated whether treatment with TPP1 or a combination of anti-GM-CSF neutralizing antibodies plus CCT137690 would antagonize Lepto1 cell viability or cancer progression. To do so, we treated Lepto1 cells for $24 \mathrm{~h}$ with DMSO (control), TPP1, anti-GM-CSF neutralizing antibodies, CCT137690, or antibodies+CCT137690 and then cultured them in conditions favoring tumorsphere formation. After 7 days, control cells developed numerous, round tumorspheres, whereas cells treated with TPP1, anti-GM-CSF antibodies, CCT137690, or antibodies+CCT137690 formed fewer and smaller tumorspheres (Suppl. Fig. 4A). CCK assays showed that every treatment also reduced the proportion of sphere-initiating cells (Suppl. Fig. 4B). 
1 However, combination treatment with anti-GM-CSF antibodies+CCT137690 reduced the proportion of live,

2 tumorsphere-initiating cells by $\sim 80 \%$ relative to DMSO treatment (Suppl. Fig. 4B), an effect that was

3 significantly greater than single drug or antibody treatment alone. To assess the effects of these treatments on

4 Lepto tumor growth, we treated Lepto1 tumorspheres with DMSO (control), TPP1, anti-GM-CSF antibodies,

5 CCT137690, or anti-GM-CSF+CCT137690 for 2 days. As expected, combination treatment significantly reduced

6 primary tumorsphere size and promoted tumorsphere dissociation (Suppl. Fig. 4A). CCK assays confirmed that

7 every treatment, and most significantly the combination treatment, reduced primary tumorsphere cell viability

8 (Suppl. Fig. 4C).

To examine the effects of each treatment on mechanisms associated with relapse, we developed secondary

tumorspheres by culturing surviving primary tumorsphere cells in standard stem cell medium without drug treatment for 12 days. Surviving cells from primary tumorspheres treated with DMSO for 2 days were dissociated and allowed to form tumorspheres, these surviving cells formed secondary tumorspheres similar to that of the primary tumorspheres (Suppl. Fig. 4A). In contrast, surviving cells from primary tumorspheres treated for 2 days with TPP1, anti-GM-CSF antibodies, CCT137690, or antibodies+CCT137690 developed fewer, more irregularlyshaped secondary tumorspheres (Suppl. Fig. 4A). The number of viable cells was also significantly lower in secondary tumorspheres derived from primary tumorspheres treated with anti-GM-CSF antibodies+CCT137690 compared to those derived from control or single-treatment primary tumorspheres (Suppl. Fig. 4D), suggesting that the combination treatment efficiently suppresses tumor regrowth. We next treated Lepto1 cells in vitro with anti-GM-CSF antibodies plus CCT137690 or Bosutinib and, in both cases, observed strong suppression of the phosphorylation-mediated activation of AKT, STAT5, and mTOR (Fig. 5G). Combination treatment with CCT137690 also significantly induced Lepto1 cell apoptosis (Suppl. Fig. 4E). Because combination treatment with antibodies+CCT137690 most potently suppressed Lepto1 cell viability and tumorsphere initiation, growth, and relapse among all of the treatments, we evaluated the effects of this combination on HER2+ LC growth in vivo. To do so, we administered anti-GM-CSF antibodies+CCT137690 on Days 5, 10, and 15 after implantating

Lepto1 cells into NOD/SCID mice (Fig. 5H). Compared to vehicle control treatment, this combination treatment 
1 significantly reduced tumor volume over time, as indicated by BLI analysis (Fig. 5I), and augmented survival 2 (Fig. 5I).

3

4

5

6

7

8

9

10

11

12

13

14

15

16

17

18

19

20

21

22

23

24

25

26 


\section{Discussion}

CNS metastases from breast cancer may involve the parenchymal brain or leptomeninges39, 54, 55, 56.

3 HER2+ breast cancer is the most common solid tumor origin of leptomeningeal metastasis $57,58,59,60,61,62,63,64$.

4 Once the tumor cells reach the leptomeninges, they may spread via the CSF65. HER2+ LC is not always 5 discernible disease based on magnetic resonance imaging (MRI), so diagnoses are made via positive cytology of 6 aspirated CSF samples. However, in some cases, adherent nodular deposits may develop on the surface of the 7 brain, spinal cord, and spinal roots, allowing diagnosis based on MRI alone14, 22, 65, 66. The presence of nodular 8 deposits is associated with the greatest suffering from headaches and intractable pain due to cranial and spinal 9 nerve invation $22,65,66$. Therefore, there is an urgent need to develop therapies that effectively target HER2+ nodules 10 in the leptomeninges. So, better understanding of the molecular mechanisms governing metastatic organotropism 11 is needed to improve precision medicine for metastatic disease.

Alternatively, various research groups have demonstrated that approximately $84 \%$ of breast cancers contain at least one genomic alteration that can be potentially exploited as a treatment target67. Indeed, genetic screens have identified promising therapeutic targets in breast cancer68. However, only a few, including phosphatidylinositol-4,5-bisphosphate 3-kinase catalytic subunit alpha (PIK3CA), AKT1, and ERBB2 8, 68, have been validated in clinical studies, suggesting that the success rate of these strategies is poor.

Despite the significant progress made over the past decade based on a combination of clinical profiling data and experimental models, our understanding of HER2+ LC remains limited, partly due to the absence of bonafide HER2+ LC in vitro and/or in vivo models. Furthermore, HER2+ LC research has been mostly based on floating HER2+ LC cells removed from the CSF in numbers insufficient to generate cell lines. Interestingly these HER2+ LC floating cells differ phenotypically from nodular tissue, which represents the major clinical burden of HER2+ LC. Therefore, one of the major limitations in the development of effective therapies for HER2+ LC is the lack of bonafide in vitro and in vivo HER2+ LC models that recapitulate the human disease.

So, to address this challenge, we developed expandable, low-passage HER2+ LC lines (Lepto1 and Lepto2) using nodular HER2+ LC tumor tissues isolated from patients. In vivo analysis of our HER2+ LC PDX mouse models showed that Lepto cells colonized the leptomeningeal surface of the brain, brain stem, and spinal 
1 cord, mimicking the spatial distribution of nodular HER2+ LC tumors in patients. Furthermore, we observed that

2 the white matter of NOD/SCID mice was relatively tumor-resistant when Lepto1 cells were implanted directly 3 into the brain stem, exhibiting $<5 \%$ tumor cell graft efficiency compared to $95 \%$ in gray matter. These 4 observations indicate that our nodular HER2+ LC-derived Lepto lines and PDX models recapitulate the human 5 disease and suggest that white matter is less hospitable to HER2+ LC metastatic colonization. Interestingly, the 6 Lepto cells extracted from our PDX models remained transcriptomically similar to pre-implanted, low-passage $7 \quad$ Lepto cells, indicating minimal or no genetic drift between pre- and post-implanted Lepto cells.

We also showed that OPCs, which are found primarily in the white matter, inhibit HER2+ LC cell viability in vitro and in vivo. Immunoblotting mediated quantification of various cytokines released into the culture media by Lepto1 cells in the presence or absence of OPCs, we identified GM-CSF to acts as an autocrine driver of Lepto cell growth in vitro and in vivo. We also demonstrated that conditional overexpression of GM-CSF in Lepto1 cells partially rescued them from OPC-induced apoptosis and suppression of viability in primary Lepto1 cells. In addition, LC-MS/MS analyses of media from Lepto1 cells cultured individually or co-cultured with either OPCs or astrocytes identified TPP1 to be secreted from OPCs and that treatment with either OPC-derived or exogeneous TPP1 abrogated GM-CSF-mediated signaling.

TPP1 is a lysosomal serine protease that also serves as a non-specific lysosomal peptidase69, 70. Although TPP1 deficiency is associated with various fatal neurodegenerative diseases $71,72,73,74,75$, such as neuronal ceroid lipofuscinoses, its role in inhibiting HER2+ LC tumor development in the white matter remains unexplored. Our studies demonstrate that OPC-secreted TPP1 plays inhibit GM-CSF-induced HER2+ LC tumor growth. We were able to also show that extracellular levels of GM-CSF drop significantly when Lepto1 cells are incubated with either OPCs or recombinant TPP1. ELISA based quantification of GM-CSF in the CSF (derived from variously treated PDX mice models) or in the Lepto1 culture media indicate OPC secreted TPP1 degrades GM-CSF, suggesting that intrathecal administration of recombinant TPP1 may be a viable therapeutic option to target HER2+ LC growth.

In addition, found that the administration of anti-GM-CSF neutralizing antibodies also abrogates GMCSF-mediated signaling and significantly impairs HER2+ LC development in vivo. To identify other potential 
1 drugs to suppress GM-CSF signaling, we performed a chemical genetics screen using a LOPAC-1280 library and

2 identified two antagonists of Lepto1 cell viability: CCT137690, a highly selective pan-Aurora kinase inhibitor,

3 and Bosutinib, an SRC tyrosine kinase inhibitor. Either drug, when combined with anti-GM-CSF neutralizing

4 antibodies, significantly inhibited the activation of downstream GM-CSF pathway effectors by suppressing their

5 phosphorylation. Subsequent studies revealed CCT137690 to be more potent than Bosutinib, with IC50 values less

6 than $100 \mathrm{nM}$; hence, our further analyses focused primarily on CCT137690. Interestingly, Aurora kinases regulate

7 key mitotic activities, including centrosome maturation, spindle assembly, chromosome segregation, and

8 cytokinesis76, 77, 78, 79, 80, 81. Indeed, Aurora kinase inhibitors have been extensively studied as novel anti-mitotic

9 drug targets82,83 and the overexpression of Aurora-A kinase has been observed in HER2+ LC tissues.

10

inactivate GM-CSF effectors but also strongly inhibit primary and secondary Lepto tumorsphere initiation,

growth, and relapse. Moreover, in PDX mouse models, combination treatment with CCT137690 and anti-GM-

CSF neutralizing antibodies inhibited Lepto1 tumor growth and augmented overall animal survival more potently

than single drug, TPP1, or anti-GM-CSF antibody treatments. These findings suggest that comparable strategies

could be used to target HER2+ LC tumors in patients, and future research is warranted to establish the optimal schedule of intrathecal chemotherapy with CCT137690 and anti-GM-CSF therapy. It is still unclear if the administration of more than one drug is superior to monotherapy; however, some reports suggest that

in vitro and in vivo. Critically, these cell lines demonstrate metastatic colonization of leptomeninges in vivo and are thus useful for establishing reliable HER2+ LC PDX models via injection into the cisterna magna. Using these cell lines, we determined that GM-CSF signaling is critical for HER2+ LC growth and that OPC-secreted TPP1 abrogates GM-CSF signaling, thus underlying the spatially heterogeneous distribution of HER2+ LC tumors in our PDX models and in patients. In addition, we found that abrogating signaling downstream of GM-CSF via the administration of TPP1, anti-GM-CSF neutralizing antibodies, or the selective pan-Aurora kinase inhibitor 
1 CCT137690 significantly decreased HER2+ LC progression in vivo and in vitro. Overall, our identified novel 2 neural niche specific crosstalk between HER2+ LC tumors and OPCs residing predominantly in the white matter. 3 We were also able to identify GM-CSF as an autocrine oncogenic driver of HER2+ LC growth in vivo via 4 induction of downstream GM-CSF signaling. Lastly, intrathecal administration of TPP1, CCT137690 and/or anti5 GM-CSF antibodies may be an excellent strategy against HER2+ LC in the clinic. 
1 Methods and Materials

2 Ethics statements

3 The use of human specimens was approved by the City of Hope (COH) Institutional Review Board (IRB;

4 protocols \#07047 and \#16015) 84, 85, 86. Written informed consent was obtained from all the patients under protocol

$5 \quad \# 07047$, and the study was conducted in accordance with the Declaration of Helsinki, institutional guidelines, and

6 all local, state, and federal regulations. All mouse studies were approved by the $\mathrm{COH}$ Institutional Animal Care

7 and Use Committee (protocol \#10044).

8

9 Reagents

10 All candidate compounds/drugs, antibodies, culture media, media supplements, and other reagents used for 11 characterization and validation experiments are listed in Resource Tables 1 and 2.

13 Derivation and culture of Lepto cell lines

14 Fresh nodular HER2+ LC specimens were acquired from consented patients who underwent surgeries to acquire 15 meningeal biopsies for pathological confirmation of HER2+ LC or to decompress localized symptomatic lesions 16 (IRB protocols \#07047 and 16015). A portion of each specimen was mechanically dissociated, and CD44+/CD2417 /EpCAM+/CD49f- cells (representing epithelial and cancer stem cell phenotypes) were sorted by fluorescence18 activated cell sorting (FACS) and maintained in an incubator at $37^{\circ} \mathrm{C}$ and $5 \% \mathrm{CO}_{2}$. Two primary cell lines, Lepto1 19 and Lepto2, were derived from two different patients. Cryobanks of low-passage Lepto1 and Lepto2 cells were 20 created using STEM-CELLBANKER Cryopreservation Media (AMSBIO) and banked in liquid nitrogen at -

$21180^{\circ} \mathrm{C}$. The isolated primary cells were cultured in human CSF (hCSF) and/or Advanced DMEM/F-12 22 (Dulbecco's Modified Eagle Medium/Ham's F-12 Nutrient Mixture; Life Technologies) with various supplements 23 (Table 2) on collagen-coated T-75 flasks, as previously described 85. 


\section{CSF collection and production}

2 hCSF samples were obtained from the ventricular space of non-cancer patients receiving ventriculoperitoneal 3 shunts (under IRB\#16015). These were mainly patients with obstructive or communicating hydrocephalus, and 4 none had infections, evidence of meningitis, multiple sclerosis, or any other known diseases. After collection, the 5 samples were centrifuged at $1,000 \mathrm{~g}\left(4^{\circ} \mathrm{C}, 15 \mathrm{~min}\right)$ to remove any cells and debris, filter-sterilized (pore size: 0.45 $6 \mu \mathrm{m}$ ), and stored at $-80^{\circ} \mathrm{C}$ until use. To condition hCSF with primary cell lines and astrocytes, cells were grown 7 in hCSF for 2 days, and then the hCSF was collected and purified by centrifugation at $4,000 \mathrm{~g}\left(15 \mathrm{~min}\right.$ at $\left.4^{\circ} \mathrm{C}\right)$.

\section{$9 \quad$ FACS sorting, differentiation, and culture of various CNS cell types}

10 Human induced pluripotent stem cell (iPSC)-derived multipotent neural progenitor cells (NPCs) were obtained

11 from EMD Millipore (Cat.\# SCC035) and propagated using ENStem-A neural expansion medium (Cat.\# 12 SCM004). The cells were differentiated to terminal neurons and oligodendrocytes using ENStem-A Neuronal 13 Differentiation Medium (Cat.\# SCM017) and human OPC Expansion Media (Cat.\# SCM107; basal medium with 14 PDGF-AA, NT3, FGF2, T3, and retinoic acid), respectively, following the supplier's recommendations. NIH15 approved H9 human embryonic stem cell (hESC)-derived OPCs were also obtained and propagated using the 16 Human Oligocyte Differentiation Kit (Cat.\# SCR600). Neurons and oligodendrocytes were differentiated from 17 these hESC-derived OPCs using EMD Millipore's Human OPC Spontaneous Differentiation Complete Media 18 (Cat. \#SCM106), according to the supplier's instructions. GFAP+ reactive astrocytes were derived from NPCs87 19 and microglia were differentiated from human iPSCs88, as described previously. The differentiated microglia, 20 oligodendrocytes, neurons, OPCs, and reactive astrocytes were purified by immunostaining with anti-CD45, anti21 GALC, anti-CD90, anti-NG2, and anti-HepaCAM antibodies, respectively, followed by FACS sorting as described40, and propagated in supplier-recommended media. To recapitulate the in vivo microenvironment, the 23 cells were grown in hCSF for various durations during the in vitro experiments. Cell morphology and differentiation status were monitored using immunofluorescence staining with anti-CD45, anti-GALC, antiCD90, anti-NG2, and anti-HepaCAM antibodies. 
2 Gene knockdown was accomplished using pools of four siRNAs targeting the same gene at different sites (Thermo

3 Fisher Scientific; listed in Resource Table 1). siRNA targeting GFP and Luciferase were used as the negative or 4 scramble controls, which should not target any mRNA in the Lepto1 cells. The transfection procedure was 5 performed according to the X-tremeGENE protocol (Sigma-Aldrich).

\section{$7 \quad$ Lentivirus production and transduction of Lepto1 lines}

8 GM-CSF open reading frame (ORF) clones were obtained from OriGene (Cat\# RC211109). The ORFs were 9 cloned into pTRE3G-ZsGreen1 vectors for Tet-On 3G tetracycline-inducible expression (Takara Biotech). 10 Lentiviruses were generated via transfection of 293T-cells (ATCC) with the packaging plasmids pMD2.G and 11 psPAX2 (Addgene). Viruses were harvested 36 and $60 \mathrm{~h}$ post-transfection, concentrated via ultracentrifugation 12 at 23,000 r.p.m. for $2 \mathrm{~h} 15 \mathrm{~min}$ at $4^{\circ} \mathrm{C}$, and reconstituted in $50 \mu \mathrm{L}$ Lepto culture medium. Constructs were titrated 13 in Lepto cells via serial dilution using ZsGreen1 (GFP) as an indicator. On Day 0, the Lepto1 cells were passaged 14 in Lepto culture medium into 24-well plates coated with collagen. On Day 1, the Lepto culture medium was 15 supplemented with Polybrene ( $8 \mu \mathrm{g} / \mathrm{mL}$, Sigma), and lentiviral infections were performed in a total volume of $16250 \mu 1$ at a multiplicity of infection (MOI) of 5. Lepto1 cultures are vulnerable to damage during spinoculation; 17 thus, static infections were performed for $12 \mathrm{~h}$, then $250 \mu \mathrm{l}$ of fresh Lepto medium was added to dilute the 18 Polybrene. Parallel wells were cultured for an additional 3 days to measure infection efficiency (percentage of 19 GFP+ DAPI cells by FACS), which ranged between 30-70\%. The GFP+ cells were FACS sorted and cultured to 20 generate single-cell Lepto subclones to be used in downstream studies.

GM-CSF and TPP1 ELISA

23 The Quantikine Human GM-CSF Immunoassay, a 3.5- or 4.5-h solid-phase ELISA, was used to measure GM24 CSF levels from Lepto1 cell culture media supernates (Human GM-CSF Quantikine ELISA Kit; R\&D Systems). 25 A TPP1 ELISA kit (Ray Biotech) was used to measure TPP1 levels in the cell culture supernates and in mouse 
1 CSF. Both ELISA-based quantification kits were used according to the manufacturers' instructions and data were

2 derived from three independent experiments.

4 Immunohistochemical and immunofluorescence analysis

5 Mouse brains were fixed in 4\% paraformaldehyde, dehydrated in 30\% sucrose, and then sectioned (10 $\mu \mathrm{m})$ and

6 mounted on microscopic slides. H\&E staining to track tumor growth and immunofluorescence staining of

7 proteins, such as GFAP, Olig2, and MBP, were conducted as previously described $84,85,86$.

\section{Cell viability and apoptosis assay}

10 Cell viability was assessed using a CellTiter-Glo Luminescent Cell Viability Assay kit, according to the 11 manufacturer's protocol. Apoptosis was measured using Annexin V-FITC or staining of phycoerythrin PE12 conjugated CD326 (EpCAM-PE). Annexin V-FITC binding was analzed by flow cytometry using an FITC signal 13 detector and EpCAM-PE staining was analyzed using a PE emission signal detector. Adherent Lepto1 cells were 14 trypsinized and washed once with FBS-containing media before incubation with Annexin V-FITC or EpCAM15 PE. attachment six-well plates (Corning). After 7 days, sphere-initiating cells were detected using the Cell Counting Kit-8 (CCK) assay, according to the manufacturer's protocol. To examine the effects of CCT137690 (100 nM), TPP1 $(100 \mathrm{ng} / \mathrm{mL})$, and anti-GM-CSF antibodies $(0.1-1 \mu \mathrm{g} / \mathrm{mL})$ on tumor growth, primary tumorspheres were cultured for 5 days and then treated for 2 days before their viability was measured using the CCK assay. DMSO was used as a negative control. To examine the effects of each treatment on tumor recurrence, primary tumorspheres were trypzinized and re-seeded without drug treatment to produce secondary tumorspheres. After 12 days of treatment, secondary tumorsphere viability was measured using the CCK assay. 


\section{Real-time PCR and western blot analysis}

3 Total RNA extraction and real-time PCR analyses were conducted as previously described. Briefly, cDNA was

4 synthesized using a cDNA synthesis kit (Table 1) with oligo dT primers, and mRNA expression levels were

5 analyzed using TaqMan-based quantitative real-time PCR with primer-dye sets (Table 1), according to the

6 manufacturer's protocols. Western blot analysis of cellular protein extracts was done using primary antibodies

7 specific to GM-CSF, GM-CSFR $\alpha$, pAKT, pmTOR, pSTAT5, tubulin, and $\beta$-actin and HRP-conjugated secondary 8 antibodies (Table 1).

\section{Luciferase assays}

11 pGL4.23[luc2/minP] luciferase reporter and pGL4.74[hRluc/TK] control vectors (Promega) were used for 12 luciferase assays. Briefly, the 500-bp putative non-coding proximal promoter region of GM-CSF (+1 to -500) 13 was cloned into the pGL4.23 luciferase vector. Dual-luciferase reporter assays were performed as described 14 previously. Lepto1 cells were transfected with pGL4.23 luciferase vector harboring GM-CSF proximal promoter 15 or GFP sequence as the negative control pGL4.74 control, according to the manufacturer's instructions. The 16 firefly and Renilla luciferase reporter activity of cell extracts was measured using the Dual-Glo Luciferase Assay 17 System (Promega) on a microplate reader, according to the manufacturer's instructions. Each treatment was 18 performed in duplicate, and the experiment was repeated three times.

\section{Chemical genetics screen}

21 Lepto1 cells were seeded in 384-well plates (500 cells per well) for $24 \mathrm{~h}$ before Library of Pharmacologically Active Compounds (LOPAC-1280) compounds were added at four concentrations each (100 nM, $200 \mathrm{nM}, 400$ $\mathrm{nM}$, and $800 \mathrm{nM}$ in $0.1 \%$ DMSO) with 2 replicates per concentration. Lepto1 cells treated with $0.1 \%$ DMSO alone served as controls and were used to create the baseline. After $72 \mathrm{~h}$, cell viability was assessed using a CellTiter-Glo cell assay. 


\section{In vivo xenografts and $\mathrm{OPC} / \mathrm{drug}$ administration}

2 To evaluate tumor growth and survival in vivo, Lepto1, Lepto2, and (MDA-MB-231) 231-BR cells (100K in 20

$3 \mu \mathrm{L}$ PBS) were transduced with mCherry and firefly luciferase (mCherry:LUC, Addgene) and injected via cisterna

4 magna puncture into cohorts of NOD/SCID mice ( $\mathrm{n}=12$ per group). At 7 and 14 days post-implantation of tumor

5 cells, the mice were intrathecally injected with either OPCs (100K or 200K in $20 \mu \mathrm{L}$ PBS), CCT137690 (100

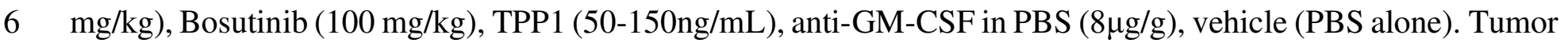

7 growth was monitored weekly using bioluminescence imaging (BLI) on a Xenogen Imaging System (Xenogen

8 Corp). At the conclusion of experiments, mice were euthanized and their brains collected, fixed in formalin

9 (Fisher Scientific), and subjected to western blot analysis.

10

\section{Statistical analyses}

12 Data are presented as mean \pm standard error. Differences between groups were evaluated using one- or two-way 13 analysis of variance (ANOVA) with Bonferroni correction for multiple comparisons (GraphPad Prism). Kaplan-

14 Meier curves were used to model overall survival. $*<0.05, * *<0.01$, and $* * *<0.001$ was considered statistically 15 significant. 
1 Acknowledgments: The authors express their gratitude to the City of Hope Analytical Cytometry Core Facility. 2 This work was made possible by the generous support of the City of Hope Department of Surgery and a grant 3 from the United States Department of Defense Breast Cancer Research Program (W81XWH-19-1-0310).

4

5 Authorship: R.J., and M.Y.C. provided the tumor biopsy samples. K.A. performed Lepto1 and Lepto2 cell line 6 derivation, subcloning, and in vivo characterization experiments and analyzed the data. A.B. performed the 7 chemical genetics screen and genetic manipulation studies and analyzed the data. A.B. wrote the manuscript. R.J., $8 \quad$ K.A., and A.B. designed and supervised the experiments and wrote the manuscript.

10 Competing interests: The authors declare no competing interests. 


\section{References}

1. Assi HI, Mahmoud T, Saadeh FS, El Darsa H. Management of leptomeningeal metastasis in breast cancer. Clin Neurol Neurosurg 172, 151-159 (2018).

2. Scott BJ, Oberheim-Bush NA, Kesari S. Leptomeningeal metastasis in breast cancer - a systematic review. Oncotarget 7, 3740-3747 (2016).

3. Niwinska A, Rudnicka H, Murawska M. Breast cancer leptomeningeal metastasis: propensity of breast cancer subtypes for leptomeninges and the analysis of factors influencing survival. Med Oncol 30, 408 (2013).

4. Tabouret E, Chinot O, Metellus P, Tallet A, Viens P, Goncalves A. Recent trends in epidemiology of brain metastases: an overview. Anticancer Res 32, 4655-4662 (2012).

5. Quigley MR, Fukui O, Chew B, Bhatia S, Karlovits S. The shifting landscape of metastatic breast cancer to the CNS. Neurosurg Rev 36, 377-382 (2013).

6. Spector R, Robert Snodgrass S, Johanson CE. A balanced view of the cerebrospinal fluid composition and functions: Focus on adult humans. Exp Neurol 273, 57-68 (2015).

7. Kennecke H, et al. Metastatic behavior of breast cancer subtypes. J Clin Oncol 28, 3271-3277 (2010).

8. Chen W, Hoffmann AD, Liu H, Liu X. Organotropism: new insights into molecular mechanisms of breast cancer metastasis. npj Precision Oncology 2, 4 (2018).

9. Nayar G, et al. Leptomeningeal disease: current diagnostic and therapeutic strategies. Oncotarget $\mathbf{8}$, $73312-73328$ (2017).

10. Jo JC, et al. Clinical features and outcome of leptomeningeal metastasis in patients with breast cancer: a single center experience. Cancer Chemother Pharmacol 72, 201-207 (2013).

11. Lin NU, Amiri-Kordestani L, Palmieri D, Liewehr DJ, Steeg PS. CNS Metastases in Breast Cancer: Old Challenge, New Frontiers. Clin Cancer Res 19, 6404-6418 (2013).

12. Jin X, Mu P. Targeting Breast Cancer Metastasis. Breast cancer : basic and clinical research 9, 23-34 (2015).

13. Lee DW, Lee KH, Kim JW, Keam B. Molecular Targeted Therapies for the Treatment of Leptomeningeal Carcinomatosis: Current Evidence and Future Directions. International journal of molecular sciences 17, (2016).

14. Leal T, Chang JE, Mehta M, Robins HI. Leptomeningeal Metastasis: Challenges in Diagnosis and Treatment. Current cancer therapy reviews 7, 319-327 (2011).

15. DeAngelis LM, Boutros D. Leptomeningeal metastasis. Cancer Invest 23, 145-154 (2005).

16. Groves MD. Leptomeningeal Disease. Neurosurg Clin N Am 22, 67-+ (2011).

17. Waki F, et al. Prognostic factors and clinical outcomes in patients with leptomeningeal metastasis from solid tumors. Journal of neuro-oncology 93, 205-212 (2009). 
18. Ashworth TR. A case of cancer in which cells similar to those in the tumors were seen in the blood after death. Aust Med J 14, 146-149 (1869).

19. Brower JV, Saha S, Rosenberg SA, Hullett CR, Robins HI. Management of leptomeningeal metastases: Prognostic factors and associated outcomes. J Clin Neurosci 27, 130-137 (2016).

20. Mack F, et al. Therapy of leptomeningeal metastasis in solid tumors. Cancer Treat Rev 43, 83-91 (2016).

21. Chowdhary S, Chamberlain M. Leptomeningeal metastases: current concepts and management guidelines. Journal of the National Comprehensive Cancer Network : JNCCN 3, 693-703 (2005).

22. Kesari S, Batchelor TT. Leptomeningeal metastases. Neurol Clin 21, 25-+ (2003).

23. Larionov AA. Current Therapies for Human Epidermal Growth Factor Receptor 2-Positive Metastatic Breast Cancer Patients. Front Oncol 8, 89-89 (2018).

24. Jaeckle KA, et al. Intrathecal treatment of neoplastic meningitis due to breast cancer with a slow-release formulation of cytarabine. Brit J Cancer 84, 157-163 (2001).

25. Chamberlain MC, Kormanik PRN. Carcinomatous meningitis secondary to breast cancer: Predictors of response to combined modality therapy. Journal of neuro-oncology 35, 55-64 (1997).

26. Park MJ. Durable Response of Leptomeningeal Metastasis of Breast Cancer to Salvage Intrathecal Etoposide After Methotrexate: A Case Report and Literature Review. Am J Case Rep 16, 524-527 (2015).

27. Niwinska A, Rudnicka H, Murawska M. Breast cancer leptomeningeal metastasis: the results of combined treatment and the comparison of methotrexate and liposomal cytarabine as intra-cerebrospinal fluid chemotherapy. Clin Breast Cancer 15, 66-72 (2015).

28. Lee JS, et al. Detection of cerebrospinal fluid tumor cells and its clinical relevance in leptomeningeal metastasis of breast cancer. Breast Cancer Res Treat 154, 339-349 (2015).

29. Fujimoto S, et al. [Radiotherapy for Alleviation of Paraparesis due to Leptomeningeal and Cauda Equina Metastasis of HER2-Positive Breast Cancer: A Case Report]. No Shinkei Geka 43, 819-823 (2015).

30. Drappatz J, Batchelor TT. Leptomeningeal neoplasms. Curr Treat Options Neurol 9, 283-293 (2007).

31. Hermann B, Hultenschmidt B, Sautter-Bihl ML. Radiotherapy of the neuroaxis for palliative treatment of leptomeningeal carcinomatosis. Strahlenther Onkol 177, 195-199 (2001).

32. Pentheroudakis G, Pavlidis N. Management of leptomeningeal malignancy. Expert Opin Pharmacother 6, 1115-1125 (2005).

33. Silvani A, et al. Neoplastic meningitis from solid tumors: a prospective clinical study in lombardia and a literature review on therapeutic approaches. J Drug Deliv 2013, 147325 (2013).

34. Li X, et al. Clinical significance of detecting CSF-derived tumor cells in breast cancer patients with leptomeningeal metastasis. Oncotarget 9, 2705-2714 (2018). 
35. Kenyon SM, Flieth TL, Algeciras-Schimnich A. Comparing the performance of CA 15-3 CSF to cytology in a cohort of patients with breast cancer leptomeningeal metastasis. Clin Biochem 58, 122-124 (2018).

36. Morikawa A, et al. Characteristics and Outcomes of Patients With Breast Cancer With Leptomeningeal Metastasis. Clin Breast Cancer 17, 23-28 (2017).

37. Yang WQ, et al. Reovirus as an experimental therapeutic for brain and leptomeningeal metastases from breast cancer. Gene Ther 11, 1579-1589 (2004).

38. Boire A, Zou Y, Shieh J, Macalinao DG, Pentsova E, Massague J. Complement Component 3 Adapts the Cerebrospinal Fluid for Leptomeningeal Metastasis. Cell 168, 1101-1113 e1113 (2017).

39. Scott BJ, Kesari S. Leptomeningeal metastases in breast cancer. Am J Cancer Res 3, 117-126 (2013).

40. Zhang Y, et al. Purification and Characterization of Progenitor and Mature Human Astrocytes Reveals Transcriptional and Functional Differences with Mouse. Neuron 89, $37-53$ (2016).

41. Sterner RM, et al. GM-CSF inhibition reduces cytokine release syndrome and neuroinflammation but enhances CAR-T cell function in xenografts. Blood 133, 697-709 (2019).

42. Patnaik MM, et al. Phase 1 study of lenzilumab, a recombinant anti-human GM-CSF antibody, for chronic myelomonocytic leukemia (CMML). Blood, (2020).

43. Rodriguez RM, et al. Signal Integration and Transcriptional Regulation of the Inflammatory Response Mediated by the GM-/M-CSF Signaling Axis in Human Monocytes. Cell Rep 29, 860-872 e865 (2019).

44. Wculek SK, Khouili SC, Priego E, Heras-Murillo I, Sancho D. Metabolic Control of Dendritic Cell Functions: Digesting Information. Front Immunol 10, 775 (2019).

45. Wlodawer A, Durell SR, Li M, Oyama H, Oda K, Dunn BM. A model of tripeptidyl-peptidase I (CLN2), a ubiquitous and highly conserved member of the sedolisin family of serine-carboxyl peptidases. BMC structural biology 3, 8 (2003).

46. Magaway C, Kim E, Jacinto E. Targeting mTOR and Metabolism in Cancer: Lessons and Innovations. Cells 8, (2019).

47. Gris-Oliver A, et al. Genetic Alterations in the PI3K/AKT Pathway and Baseline AKT Activity Define AKT Inhibitor Sensitivity in Breast Cancer Patient-derived Xenografts. Clin Cancer Res, (2020).

48. Hyman DM, et al. AKT Inhibition in Solid Tumors With AKT1 Mutations. J Clin Oncol 35, 2251-2259 (2017).

49. He J, et al. Multi-targeted kinase inhibition alleviates mTOR inhibitor resistance in triple-negative breast cancer. Breast Cancer Res Treat 178, 263-274 (2019).

50. Presti D, Quaquarini E. The PI3K/AKT/mTOR and CDK4/6 Pathways in Endocrine Resistant HR+/HER2- Metastatic Breast Cancer: Biological Mechanisms and New Treatments. Cancers (Basel) 11, (2019). 
51. Hung LY, et al. Nuclear epidermal growth factor receptor (EGFR) interacts with signal transducer and activator of transcription 5 (STAT5) in activating Aurora-A gene expression. Nucleic Acids Res 36, 4337-4351 (2008).

52. Jiao Q, Bi L, Ren Y, Song S, Wang Q, Wang Y-s. Advances in studies of tyrosine kinase inhibitors and their acquired resistance. Molecular Cancer 17, 36 (2018).

53. Katsha A, Belkhiri A, Goff L, El-Rifai W. Aurora kinase A in gastrointestinal cancers: time to target. Molecular Cancer 14, 106 (2015).

54. Chen TW, et al. Systemic treatment of breast cancer with leptomeningeal metastases using bevacizumab, etoposide and cisplatin (BEEP regimen) significantly improves overall survival. Journal of neuro-oncology 148, 165-172 (2020).

55. Kumthekar P, et al. ANG1005, a Brain-Penetrating Peptide-Drug Conjugate, Shows Activity in Patients with Breast Cancer with Leptomeningeal Carcinomatosis and Recurrent Brain Metastases. Clin Cancer Res 26, 2789-2799 (2020).

56. Shigekawa T, et al. Successful treatment of leptomeningeal metastases from breast cancer using the combination of trastuzumab and capecitabine: a case report. Breast Cancer 16, 88-92 (2009).

57. Angus L, Martens JWM, van den Bent MJ, Sillevis Smitt PAE, Sleijfer S, Jager A. Novel methods to diagnose leptomeningeal metastases in breast cancer. Neuro Oncol 21, 428-439 (2019).

58. Cuppari L, Reccia P, Burei M, Cervino AR, Evangelista L. 18F-Choline PET/CT in Leptomeningeal Breast Cancer Metastases. Clin Nucl Med 44, e96-e97 (2019).

59. Ha B, et al. Effects of Postoperative Radiotherapy on Leptomeningeal Carcinomatosis or Dural Metastasis after Resection of Brain Metastases in Breast Cancer Patients. Cancer Res Treat 49, 748-758 (2017).

60. Jung JM, Kim S, Joo J, Shin KH, Gwak HS, Lee SH. Incidence and risk factors for leptomeningeal carcinomatosis in breast cancer patients with parenchymal brain metastases. J Korean Neurosurg Soc 52, 193-199 (2012).

61. Kaidar-Person $\mathrm{O}$, et al. The incidence and predictive factors for leptomeningeal spread after stereotactic radiation for breast cancer brain metastases. Breast $J$ 24, 424-425 (2018).

62. Pan Z, et al. 'Hot cross bun' sign with leptomeningeal metastases of breast cancer: a case report and review of the literature. World J Surg Oncol 13, 43 (2015).

63. Ricciardi GRR, et al. Efficacy of T-DM1 for leptomeningeal and brain metastases in a HER2 positive metastatic breast cancer patient: new directions for systemic therapy - a case report and literature review. BMC Cancer 18, 97 (2018).

64. Tham YL, Hinckley L, Teh BS, Elledge R. Long-term clinical response in leptomeningeal metastases from breast cancer treated with capecitabine monotherapy: a case report. Clin Breast Cancer 7, 164-166 (2006).

65. Chamberlain MC. Leptomeningeal metastasis. Current opinion in oncology 22, 627-635 (2010). 
66. Lin $\mathrm{N}$, et al. Benefit of ventriculoperitoneal cerebrospinal fluid shunting and intrathecal chemotherapy in neoplastic meningitis: a retrospective, case-controlled study Clinical article. J Neurosurg 115, 730736 (2011).

67. Vasan N, et al. A targeted next-generation sequencing assay detects a high frequency of therapeutically targetable alterations in primary and metastatic breast cancers: implications for clinical practice.

Oncologist 19, 453-458 (2014).

68. Onesti CE, Vicier C, André F. What to expect from high throughput genomics in metastatic breast cancers? The Breast 24, S19-S22 (2015).

69. Haney MJ, Klyachko NL, Harrison EB, Zhao Y, Kabanov AV, Batrakova EV. TPP1 Delivery to Lysosomes with Extracellular Vesicles and their Enhanced Brain Distribution in the Animal Model of Batten Disease. Adv Healthc Mater 8, e1801271 (2019).

70. Wiemhoefer A, et al. Tripeptidyl Peptidase II Mediates Levels of Nuclear Phosphorylated ERK1 and ERK2. Mol Cell Proteomics 14, 2177-2193 (2015).

71. Collier AM, et al. Lysosomal protein thermal stability does not correlate with cellular half-life: global observations and a case study of tripeptidyl-peptidase 1. Biochem J 477, 727-745 (2020).

72. Johnson TB, Cain JT, White KA, Ramirez-Montealegre D, Pearce DA, Weimer JM. Therapeutic landscape for Batten disease: current treatments and future prospects. Nat Rev Neurol 15, 161-178 (2019).

73. Lukacs Z, et al. Validity of a rapid and simple fluorometric tripeptidyl peptidase 1 (TPP1) assay using dried blood specimens to diagnose CLN2 disease. Clin Chim Acta 492, 69-71 (2019).

74. Smith PK, Sen MG, Fisher PR, Annesley SJ. Modelling of Neuronal Ceroid Lipofuscinosis Type 2 in Dictyostelium discoideum Suggests That Cytopathological Outcomes Result from Altered TOR Signalling. Cells 8, (2019).

75. Wong LC, Hsu CJ, Lee WT. Perampanel attenuates myoclonus in a patient with neuronal ceroid lipofuscinoses type 2 disease. Brain Dev 41, 817-819 (2019).

76. Dhanasekaran K, et al. Unraveling the role of aurora A beyond centrosomes and spindle assembly: implications in muscle differentiation. FASEB J 33, 219-230 (2019).

77. Fielding AB, Dobreva I, Dedhar S. Beyond focal adhesions: integrin-linked kinase associates with tubulin and regulates mitotic spindle organization. Cell Cycle 7, 1899-1906 (2008).

78. Kobayashi A, Hashizume C, Dowaki T, Wong RW. Therapeutic potential of mitotic interaction between the nucleoporin Tpr and aurora kinase A. Cell Cycle 14, 1447-1458 (2015).

79. Magnaghi-Jaulin L, Eot-Houllier G, Gallaud E, Giet R. Aurora A Protein Kinase: To the Centrosome and Beyond. Biomolecules 9, (2019).

80. Mielgo A, et al. A MEK-independent role for CRAF in mitosis and tumor progression. Nat Med 17, 1641-1645 (2011).

81. So C, et al. A liquid-like spindle domain promotes acentrosomal spindle assembly in mammalian oocytes. Science 364, (2019). 
82. Faisal A, et al. The aurora kinase inhibitor CCT137690 downregulates MYCN and sensitizes MYCNamplified neuroblastoma in vivo. Mol Cancer Ther 10, 2115-2123 (2011).

83. Cirak Y, Furuncuoglu Y, Yapicier O, Aksu A, Cubukcu E. Aurora A overexpression in breast cancer patients induces taxane resistance and results in worse prognosis. J BUON 20, 1414-1419 (2015).

84. Ansari KI, Bhan A, Liu X, Chen MY, Jandial R. Astrocytic IGFBP2 and CHI3L1 in cerebrospinal fluid drive cortical metastasis of HER2+breast cancer. Clin Exp Metastasis 37, 401-412 (2020).

85. Choy C, et al. Cooperation of neurotrophin receptor TrkB and Her2 in breast cancer cells facilitates brain metastases. Breast Cancer Res 19, 51 (2017).

86. Jandial R, Choy C, Levy DM, Chen MY, Ansari KI. Astrocyte-induced Reelin expression drives proliferation of Her2(+) breast cancer metastases. Clin Exp Metastasis 34, 185-196 (2017).

87. Neman J, et al. Co-evolution of breast-to-brain metastasis and neural progenitor cells. Clin Exp Metastasis 30, 753-768 (2013).

88. Pandya $\mathrm{H}$, et al. Differentiation of human and murine induced pluripotent stem cells to microglia-like cells. Nat Neurosci 20, 753-759 (2017). 
Figure 1. Derivation and characterization of nodular HER2+ LC cells.

A

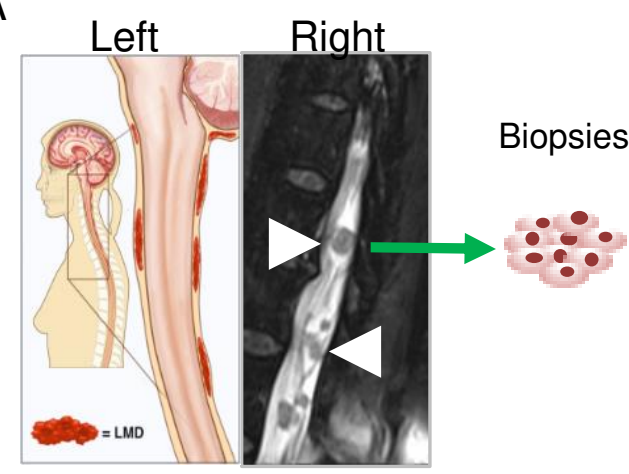

B

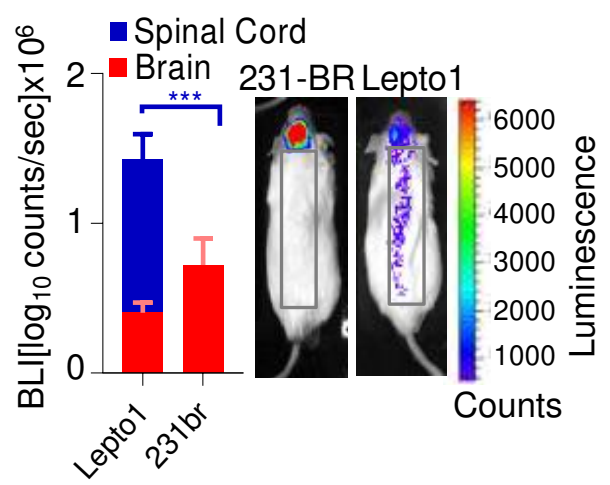

C

E

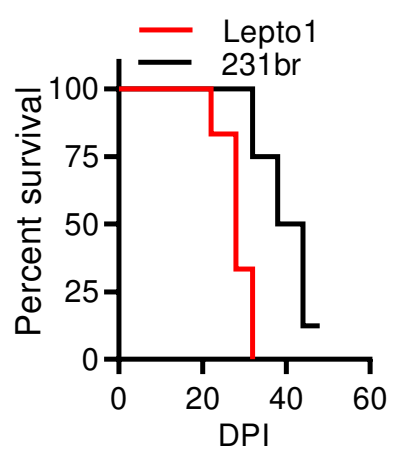

D

$\mathrm{F}$
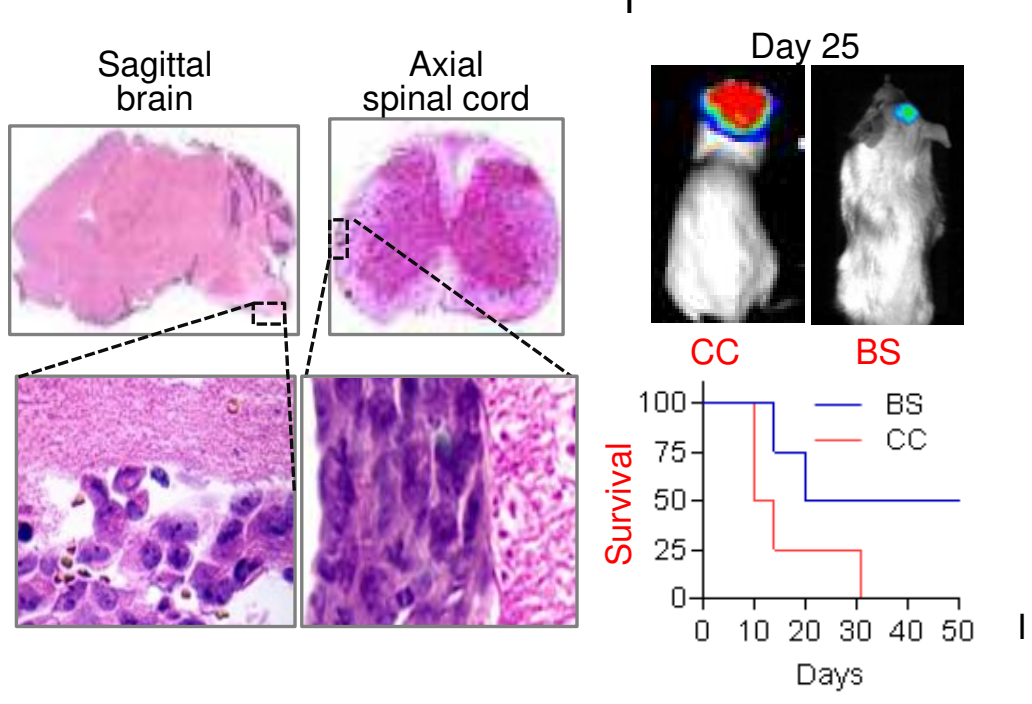

G

$\mathrm{H}$
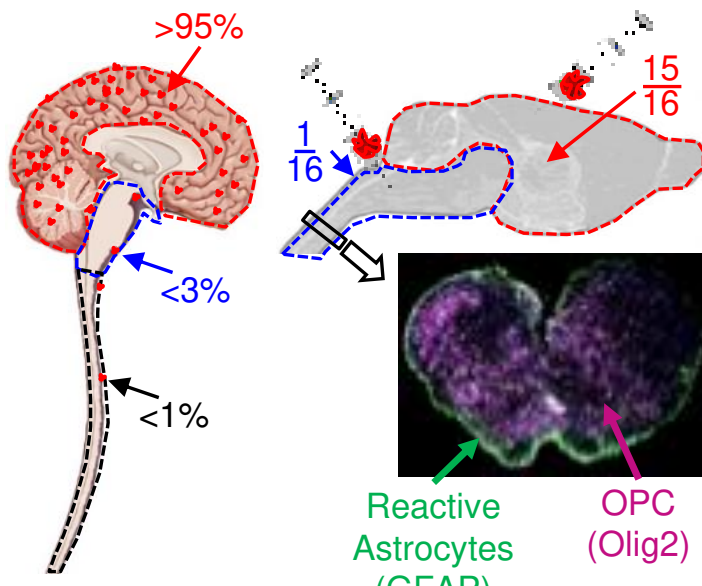

Reactive

Astrocytes (GFAP)

Functional

Characterization
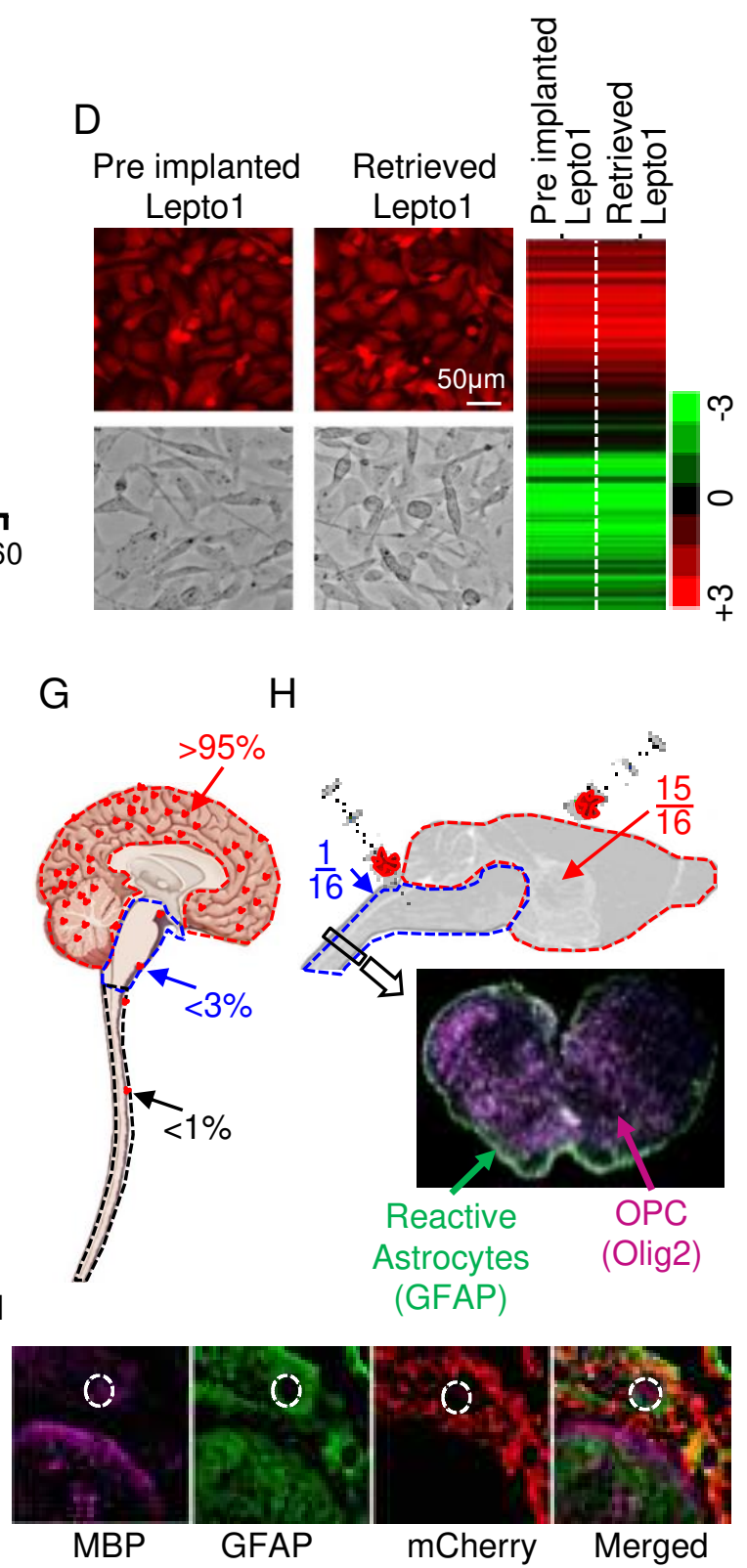
1 A.) Schematic (left) and T2-weighted sagittal MRI of the spine (right) showing the distribution of nodular HER2+

2 LC tumors in a breast cancer patient. White arrows indicate nodular deposits. Also shown are steps taken from 3 the derivation of our Lepto lines to their functional characterization.

4 B.) BLI image and quantification of brain-tropic breast cancer (231-BR) and HER2+ LC (Lepto1) cells in the 5 brain and spinal cord of PDX models. $* * *<0.001$.

6 C.) Kaplan-Meier survival analyses in days post-implantation (DPI) showing shorter survival of mice bearing $7 \quad$ Lepto1 compared to mice bearing $231 \mathrm{br}$ cells.

8 D.) (Top row) Immunofluorescence images of mCherry: LUC-labeled Lepto1 Her2+ LC (Lepto1 lines) cells in NOD/SCID mice before injection (pre-implanted, left panels) and 24 days after (retrieved, center panels). (Bottom row) The cells exhibited comparable morphology based on brightfield images (scale bar $=50 \mu \mathrm{m}$ ). (Right panels) Transcriptomic analysis of pre-implanted and retrieved Lepto1 cells revealed comparable transcriptional signatures.

E.) (Top panel) Histopathological analysis of the sagittal section of the brain and axial spinal cord showing Lepto1 deposition only in the leptomeninges of CNS without invasion of the spinal cord parenchyma. (Bottom panel) $\mathrm{H} \& \mathrm{E}$ staining at higher magnification showing the presence of Lepto1 cells in the leptomeninges.

F.) (Top panel) Representative BLI images of Lepto1 cells implanted into the white matter of the brain stem (BS) and the gray matter of cerebral cortex (CC) of PDX models, shown at Day 25. CC-implanted mice developed larger metastatic tumor deposits. (Bottom panel) Kaplan-Meier survival analyses showing shorter survival of mice bearing Lepto1 cells in the CC, as compared to mice bearing Lepto1 cells in the BS.

G.) Schematic showing the heterogeneous distribution of metastatic HER2+ LC tumors in patients.

H.) (Top panel) Schematic showing the grafting efficiency of HER2+ LC tumor-derived Lepto1 cells injected into the spinal cord of NOD/SCID mice (1 per 16 injections) versus the cerebral cortex (15 per 16 injections). (Bottom panel) Immunohistochemistry of the mouse spinal cord showing the distribution of reactive astrocytes (GFAP+, green) and OPCs (Olig2+, purple) following injection of HER2+ LC tumor-derived Lepto1 cells into the spinal cord.

I.) Immunofluorescence analysis of serial spinal cord sections taken from mice 10 days after Lepto1 cell implantation. Staining with markers of oligodendrocytes (MBP, purple), astrocytes (GFAP, green), and mCherry (Lepto cells, red) shows a GFAP+ layer of reactive astrocytes on the surface of the brain stem and spinal cord juxtaposed to nodular HER2+ LC. 
Figure 2. The presence of OPCs reduces HER2+ LC cell viability.

B

C
A

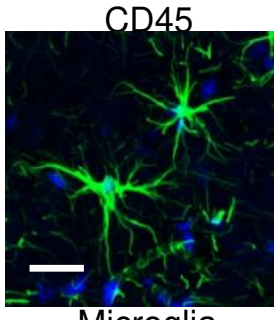

Microglia

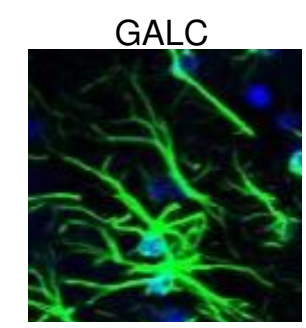

Oligodendrocyte

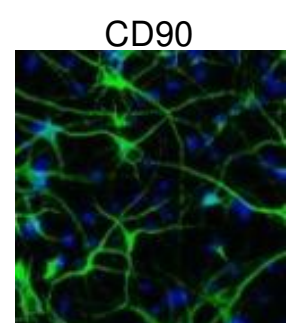

Neuron

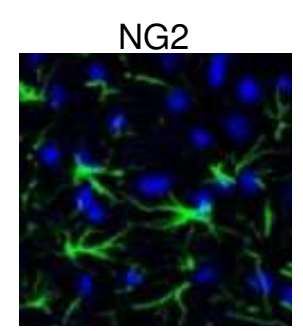

OPC

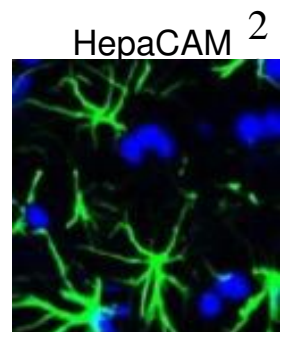

Astrocytes

DAPI Cell-type Specific markers
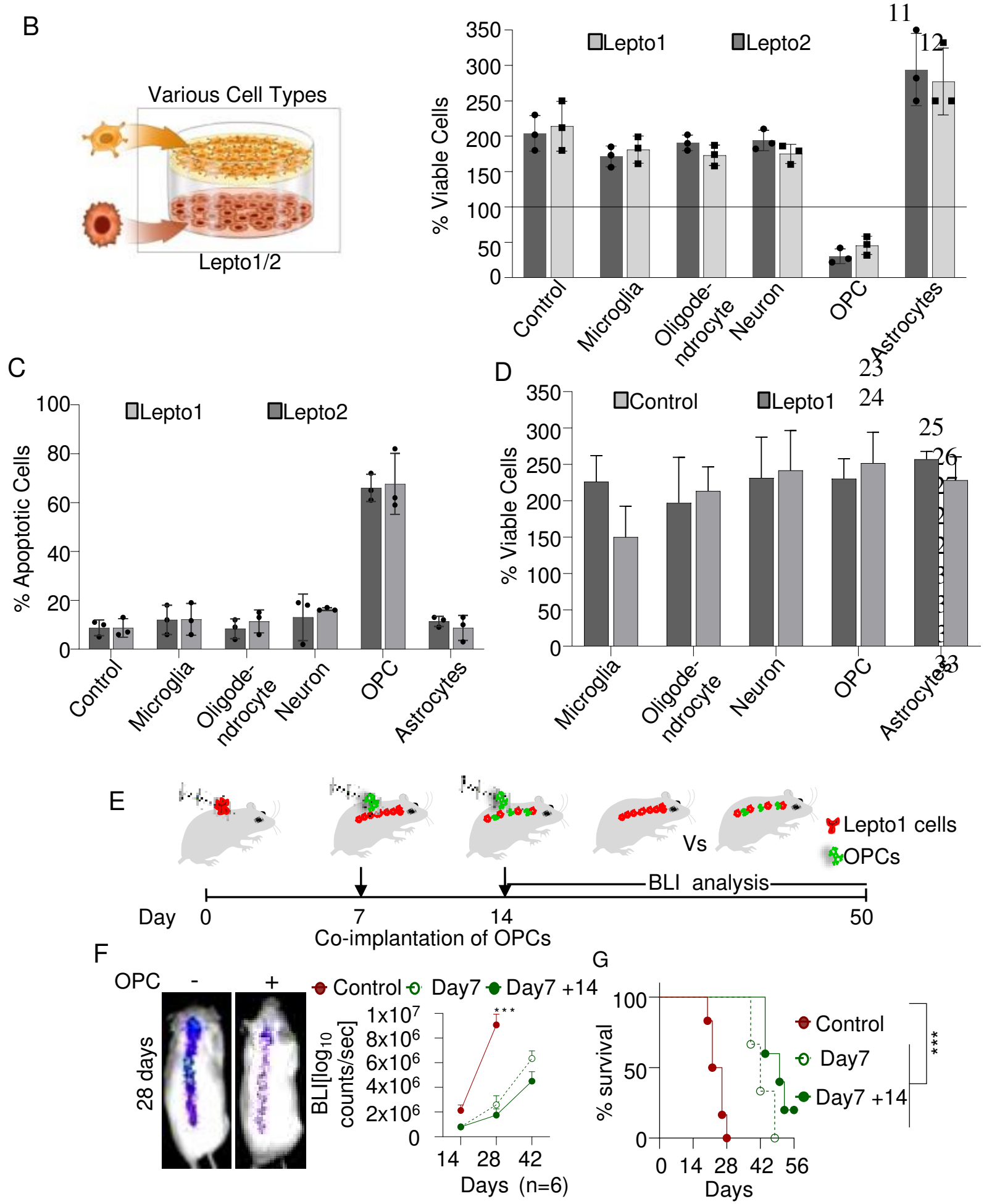

Lepto1 cells oPCs

G

14

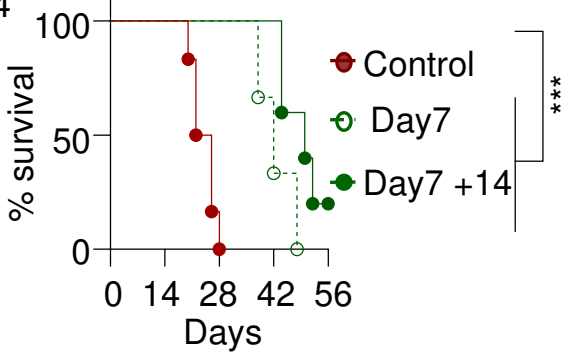


2 A.) Immunofluorescence analyses of various CNS cell types immuno-panned from human iPSCs and stained with 3 the indicated antibodies. Scale bar=100 $\mu \mathrm{m}$.

4 B.) (Left panel) Schematic showing the Boyden chamber system used to evaluate the effects of various CNS cell types on the viability of Lepto1 and Lepto2 cell lines. (Right panel) Viability analyses of Lepto1 and Lepto2 cell lines that were co-cultured with the various CNS cell types (derived in figure 2A) for $48 \mathrm{~h}$, using CellTiterGlo. $n=3$.

C.) Annexin V FACS-based analysis of Lepto1 and Lepto2 cells co-cultured with the indicated human CNS cell populations. Co-culture with OPCs increased the proportion of apoptotic Lepto cells, $\mathrm{n}=3$.

D.) Viability analyses based on CellTiter-Glo assays of various CNS cell types (derived in 2A) co-cultured with Lepto1 lines for $48 \mathrm{~h}, \mathrm{n}=3$

E.) Schematic showing the protocol used for the in vivo characterization of the effects of OPCs on Lepto1 cell growth. mCherry: LUC-labeled Lepto1 cells (100K, red) were injected into the cisternae magna of adult NOD/SCID mice on Day 0, and OPCs (100K, green) were injected on Days 7 and/or 14. Representative BLI images are from Day 28.

F.) (Left panel) Representative BLI images of mice on Day 28 of the experiment illustrated in $2 \mathrm{E}$, with and without co-implantated OPCs. (Right panel) Quantitative analysis revealed that mice that received OPCs exhibited reduced tumor growth $(\mathrm{n}=6)$. ***p<0.001 relative to OPC implanted on D7+D14.

G.) Kaplan-Meier based overall survival analysis and comparison of mice implanted with Lepto1 cells only on D0 (solid red line) or co-implanted with Lepto1 cells on D0 along with OPCs on D7 or with OPCs implanted on D7 as well as on D14 (solid green), *** p<0.001 relative to vehicle-treated controls. 
2 Figure 3. GM-CSF acts as an oncogenic autocrine driver of HER2+ LC cell growth.

A

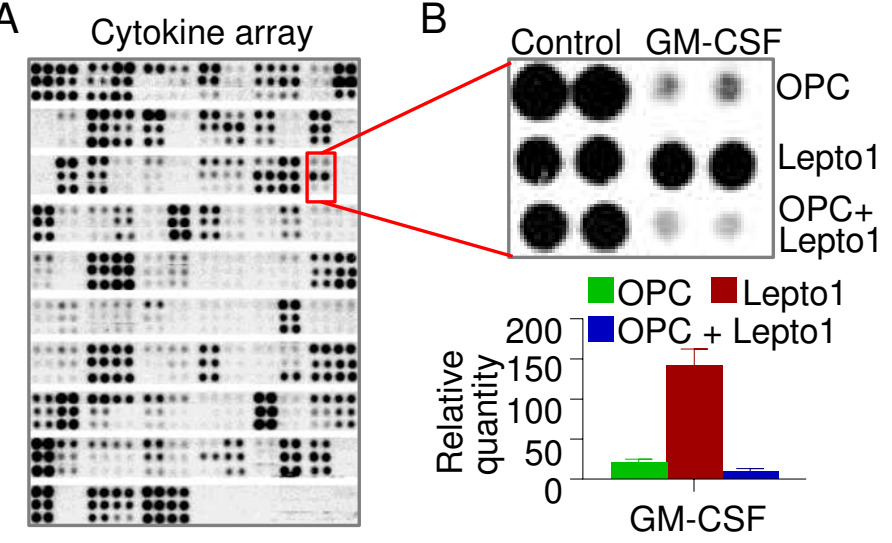

C

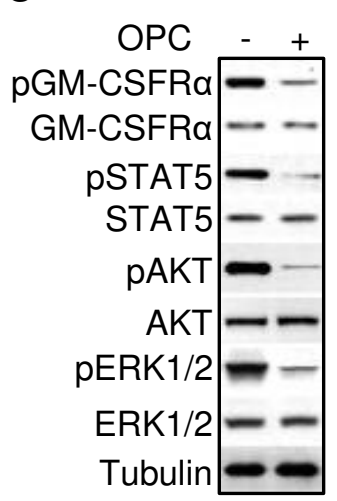

4

pGM-CSFRa

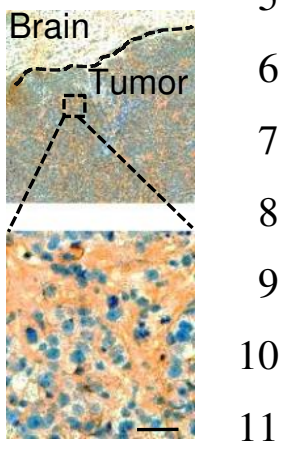

12

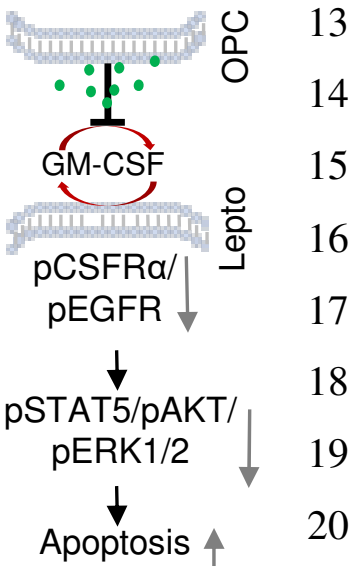

21

18

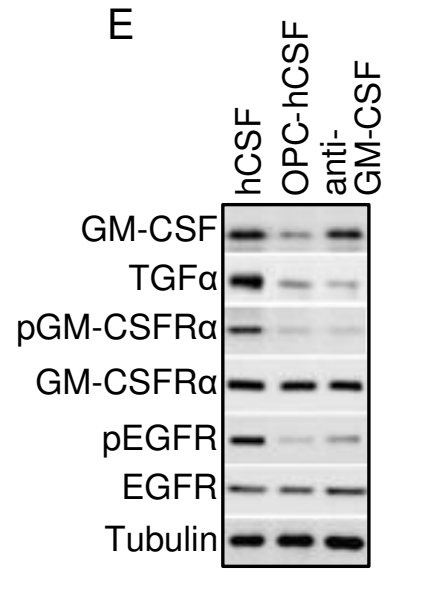

F

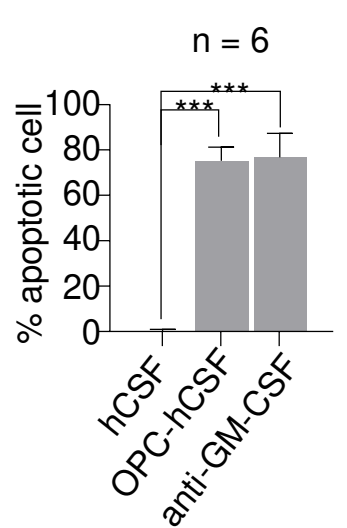

$\mathrm{H}$
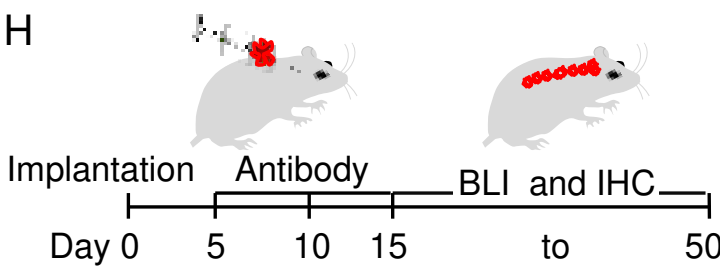

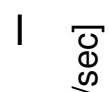

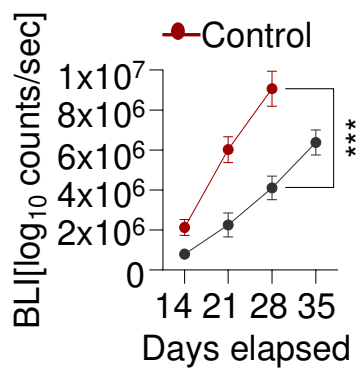

- Anti-GM-CSF

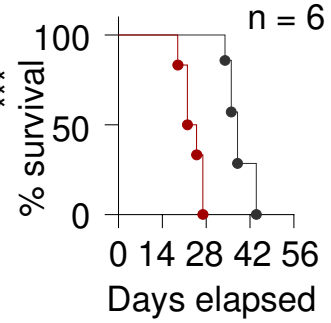

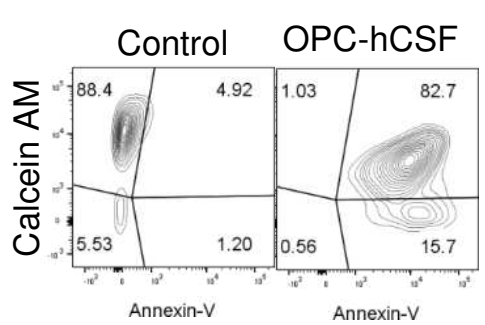

Anti-GM-CSF

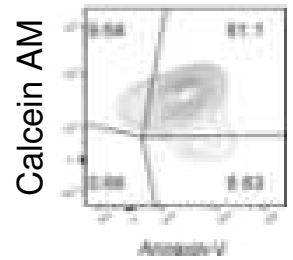

K

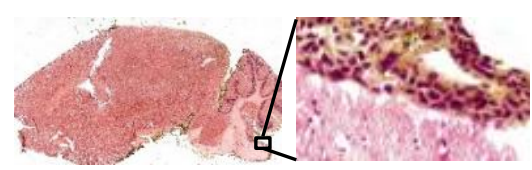
Control lgG

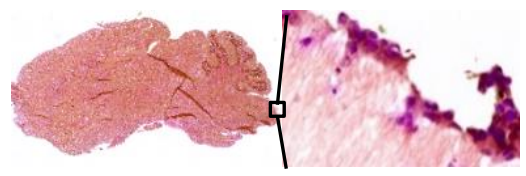

Anti-GM-CSF IgG

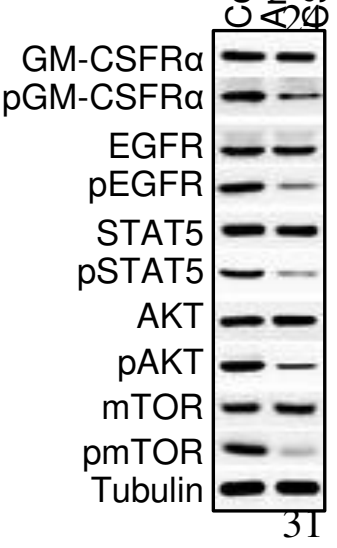


1 A.) Cytokine XL array-based analyses of conditioned media from OPCs and/or Lepto1 cells cultured in media supplemented with hCSF. Secreted factors identified in these analyses are listed in Supplementary Table 1.

3 B.) (Top panel) Representative portion of the array blot described in 3A. (Bottom panel) Density-based 4 quantification of blots shown in Figure 3A.

5 C.) Western blot analysis of signaling proteins in extracts from Lepto1 cells cultured alone or with OPCs for 48
h. The analysis shows lower pGM-CSFR $\alpha$ levels and less growth factor phosphorylation/actiavation (pSTAT5, pAKT, and pERK1/2) in co-cultured Lepto1 cells.

D.) Immunohistochemical analysis of patient HER2+ LC specimens showing pGM-CSFR $\alpha$ (orange) in tumor cells but not surrounding brain tissue. Scale bar $=100 \mu \mathrm{m}$.

E.) Western blot analysis of the indicated signaling factors associated with the GM-CSF pathway in Lepto1 cells grown in hCSF only, OPC-conditioned hCSF, or hCSF containing anti-GM-CSF neutralizing antibodies. GMCSF signaling was disrupted in samples grown in OPC-conditioned media and anti-GM-CSF neutralizing antibodies. Tubulin served as a loading control.

F.) (Left panel) Quantification of Annexin V-positive Lepto1 cells grown under the indicated conditions. Cells cultured with OPC-conditioned media or anti-GM-CSF neutralizing antibodies were significantly more apoptotic than control cells. (Right panel) Representative FACS plots demonstrating augmented Annexin V (pro-apoptotic) staining of live (Calcein Blue AM-positive) Lepto1 cells assessed in the left panel.

G.) Schematic showing the pathway through which GM-CSF induces HER2+ LC growth and how OPCs may block that pathway.

H.) Schematic showing the protocol used for the implantation of mCherry:LUC-labeled Lepto1 cells (100K) into mice, followed by the intrathecal administration of anti-GM-CSF neutralizing or control IgG antibodies on Days 5, 10, and 15 .

I.) (Left panel) BLI-based analysis of mCherry:LUC-labeled Lepto1 tumors in mice treated with anti-GM-CSF antibodies or control IgG. Antibody treatment reduced tumor progression. (Right panel) Survival analysis of mice treated as shown in Figure $3 \mathrm{H}(\mathrm{n}=6) . * * * \mathrm{p}<0.001$.

J.) Immunohistochemical analysis of brain sections from Lepto1-implanted NOD/SCID mice treated with antiGM-CSF antibodies or control IgG. Anti-GM-CSF antibody treatment suppressed Lepto1 growth.

K.) Western blot analysis of the indicated signaling factors in HER2+ LC PDX tumors isolated from mice that received intrathecal anti-GM-CSF antibodies or control IgG. Antibody treatment decreased the phosphorylation of GM-CSFR $\alpha$, EGFR, and various growth factors (STAT5, AKT, and ERK1/2). 
Figure 4. OPC-derived TPP1 is a candidate regulator of GM-CSF in HER2+ LC cells.

A

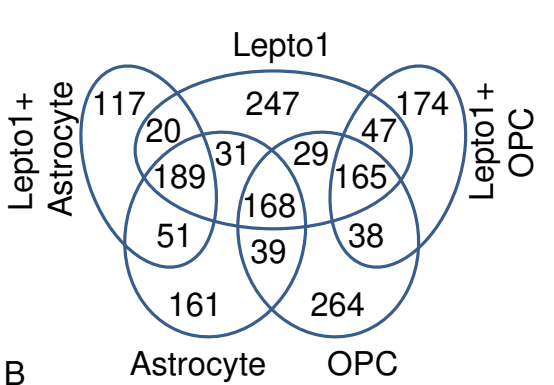

B

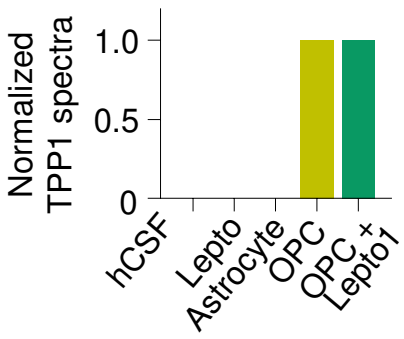

G

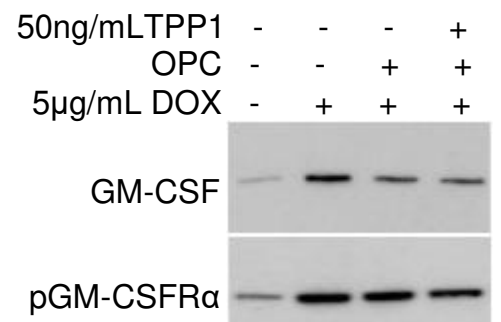

$\mathrm{H}$
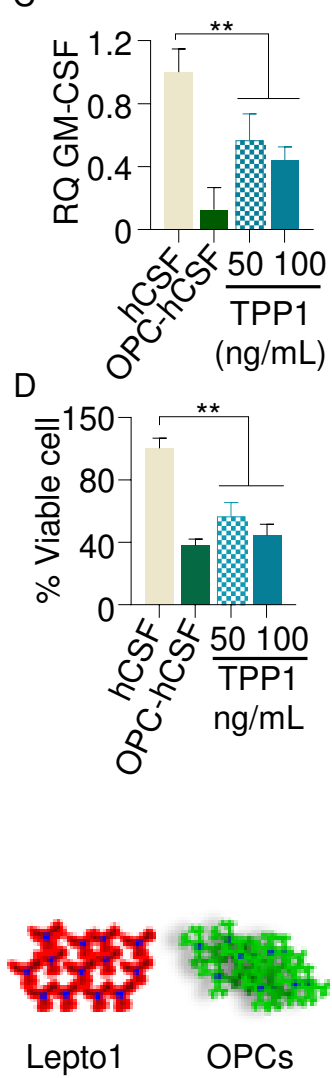

E

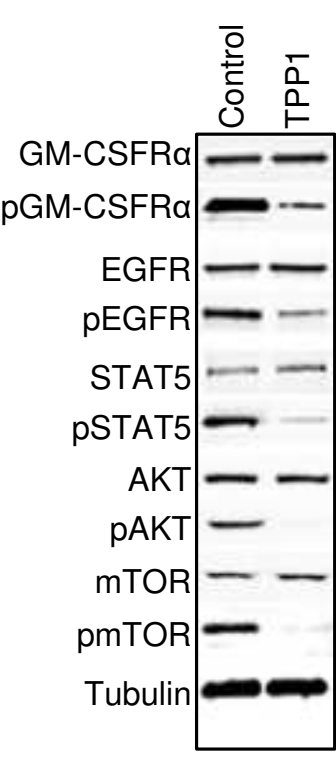

F 5

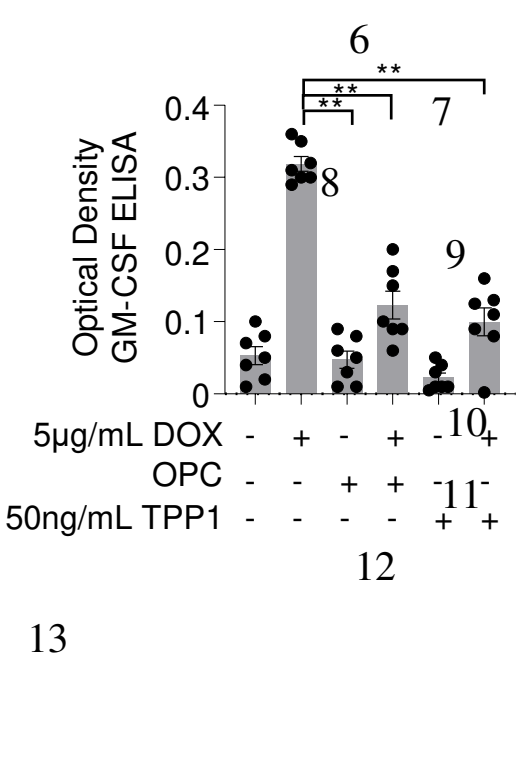

15
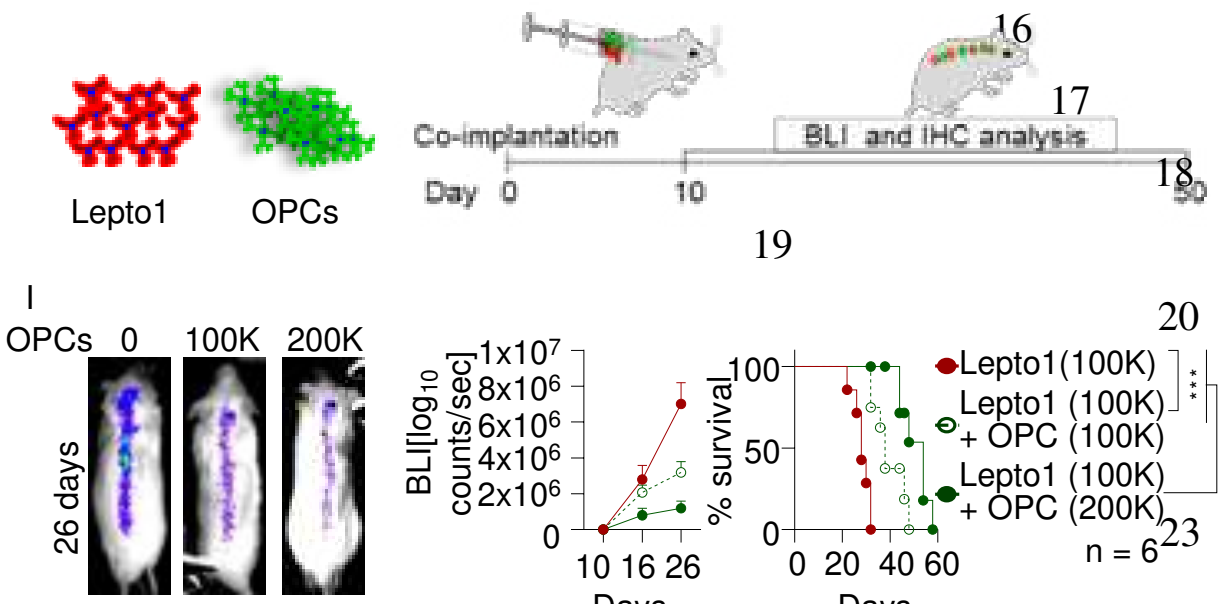

20
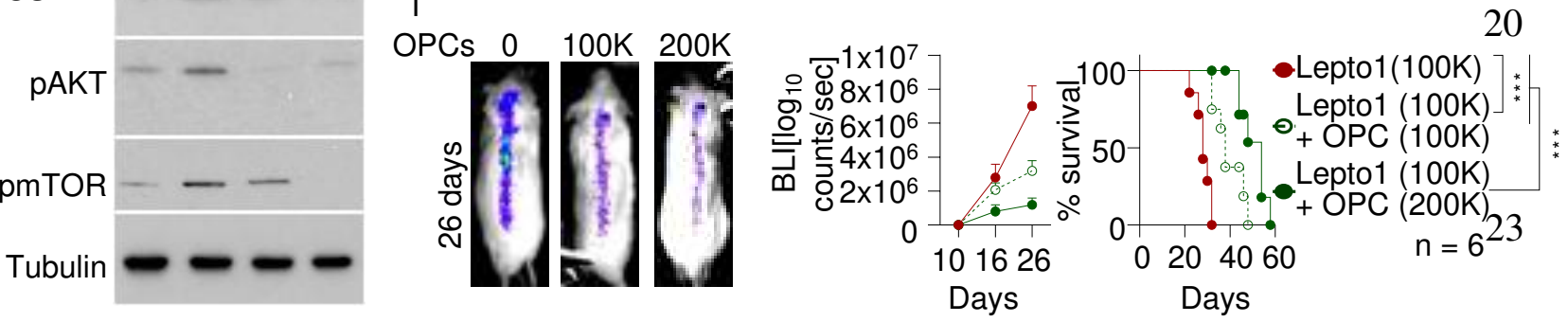
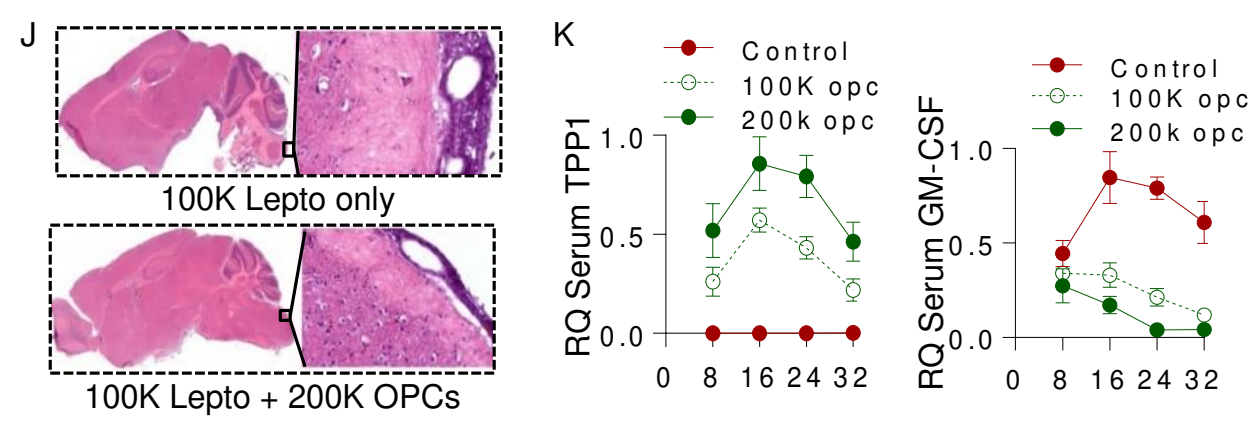
2 A.) Venn diagram of unique and shared extracellular proteins identified in the hCSF of cultured astrocytes, OPCs, 3 and Lepto1 cells alone or in the indicated co-cultures.

4 B.) Relative TPP1 protein levels in control hCSF media (no cells) and hCSF from the indicated cell cultures.

5 C.) Relative quantification of GM-CSF levels in media from Lepto1 cells cultured in OPC-conditioned hCSF or $6 \quad$ with exogenous TPP1 (50 or $100 \mathrm{ng} / \mathrm{mL}$ ), as measured by ELISA. ** $\mathrm{p}<0.01$.

7 D.) Lepto1 cell viability, as measured by a CellTiter-Glo assay, following treatment with OPC-conditioned hCSF 8 or exogenous TPP1, as shown in Figure 4C. $* * \mathrm{p}<0.01$.

9 E.) Western blot analysis of Lepto1 cells to assess the phosphorylation status of the indicated downstream effectors of GM-CSF in untreated (control) cells or cells treated with exogenous TPP1 (100 ng/mL). TPP1 treatment reduced phosphorylation and hence activation of STAT5, AKT, and mTOR without altering total protein levels.

F.) ELISA analysis of GM-CSF in the media of Lepto1 cells conditionally overexpressing GM-CSF and cultured with OPCs or TPP1 (50 ng/mL). Both treatments reduced levels of GM-CSF in culture media. ** $\mathrm{p}<0.01$.

G.) Western blot analysis of downstream effectors of GM-CSF and GM-CSFR $\alpha$ in Lepto1 cells conditionally overexpressing GM-CSF that were co-cultured with OPCs, with or without TPP1 $(50 \mathrm{ng} / \mathrm{mL})$, indicating that both OPC and TPP1 suppress phosphorylation of GM-CSFR $\alpha$ and its downstream targets, AKT and mTOR.

H.) Schematic showing the protocol used for the co-implantation of Lepto1 cells and OPCs at various cell densities $(0,100 \mathrm{~K}$, and $200 \mathrm{~K})$.

I.) (Left panel) Representative BLI images of NOD/SCID mice on Day 26 post-implantation of Lepto1 cells alone or with OPCs $(100 \mathrm{~K}$ or $200 \mathrm{~K})$. (Center panel) BLI-based quantification of tumor growth and (Right panel) Kaplan-Meier curves showing OPC density-dependent suppression of Lepto1 tumor progression and enhancement of animal survival. $* * * \mathrm{p}<0.001$.

J.) H\&E staining of brain tissue sections from NOD/SCID mice implanted with Lepto cells only (100K) or with Lepto1 (100K) and OPC cells (200K).

K.) TPP1 and GM-CSF levels were measured in CSF extracted from the mice implanted with Lepto1 cells or OPCs on days 8, 16, 24, and 32. ELISA based analyses of TPP1 (left) and GM-CSF (right) in serum collected from mice implanted with Lepto cells only (100K, Control) or with Lepto1 cells $(100 \mathrm{~K})$ and OPCs (100K or 200K). TPP1 levels increase and GM-CSF levels decrease with increasing OPC cell density. 
1 Figure 5. Combination treatment with a pan-Aurora kinase inhibitor and anti-GM-CSF neutralizing 2 antibodies antagonizes Lepto cell growth in vivo.

A

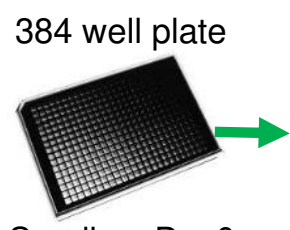

Seeding; Day0

\section{LOPAC1280}

100nM, 200nM, 400nM, 800nM

Library addition;

Day1
CellTiterGlo Viability assay Viability screen; Day4

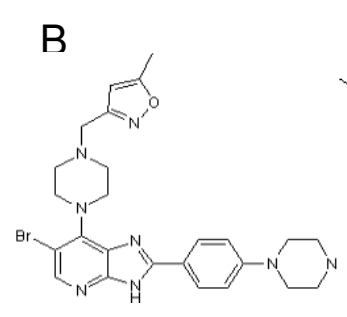

CCT137690
3

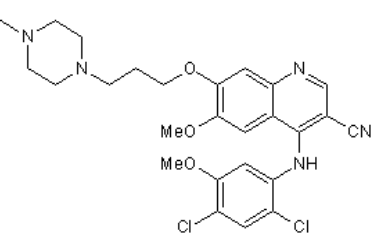

Bosutinib

5

C

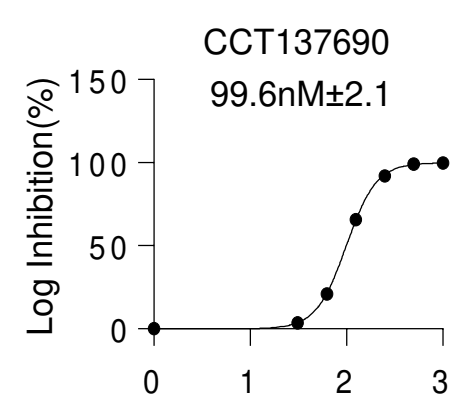

Concentration $\left(\log _{10}\right)$

6

E

AURKA GM-CSF SRO

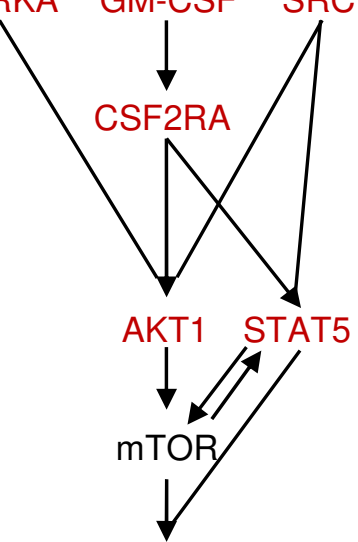

Downstream targets

Cell proliferatiøn

16
Bosutinib (SRC)

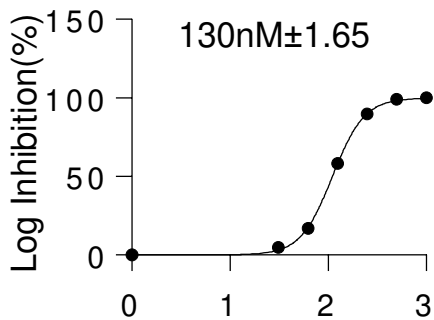

Concentration $\left(\log _{10}\right)$

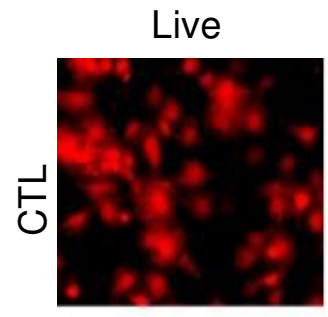

Apoptotic
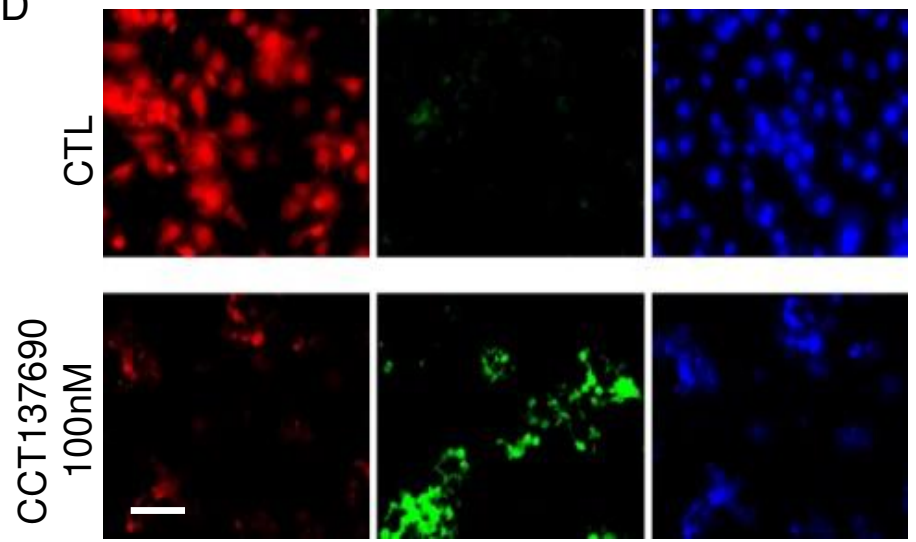

8

G
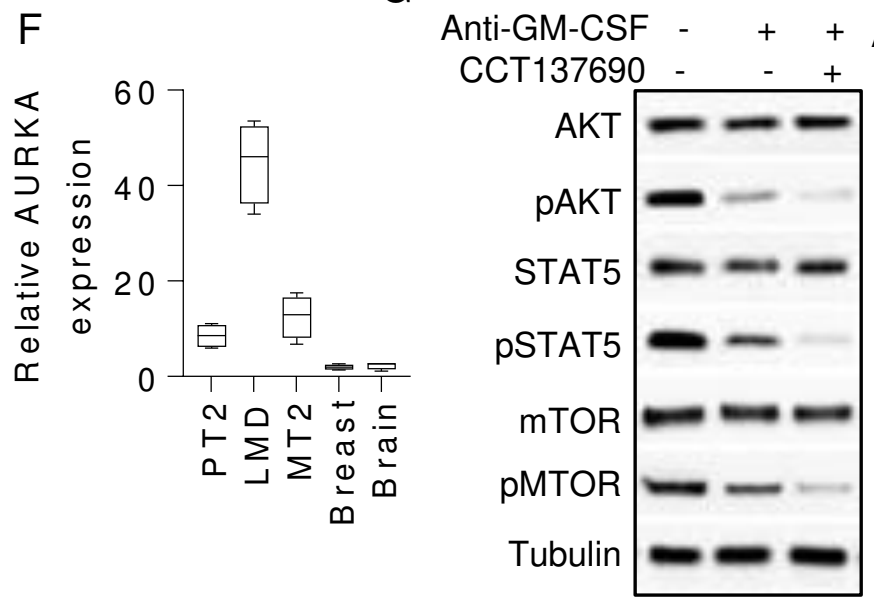

nti-GM-CSF Bosutinib
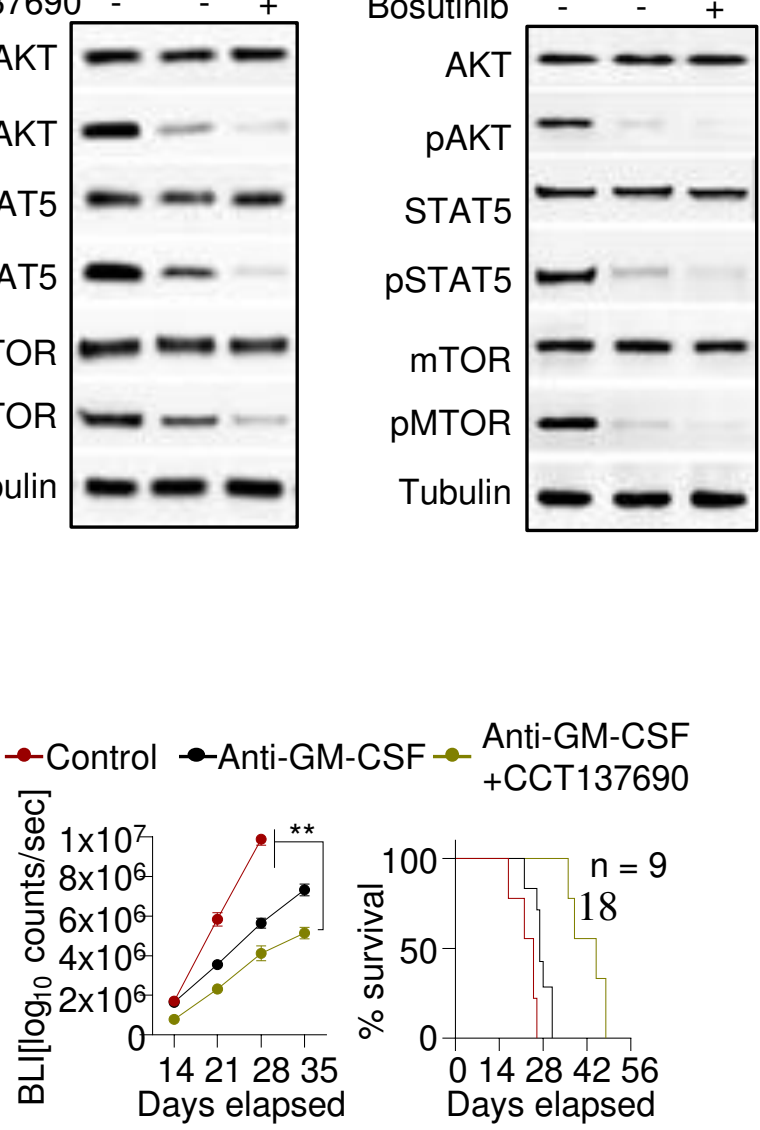
2 A.) Schematic showing the submicromolar chemical genetics screen performed using the LOPAC-1280 library. $3 \sim 1,000$ Lepto1 cells were seeded on 384-well plates on Day 0 and treated as shown.

4 B.) Chemical structures of the top two hits identified in the primary screen described in 5A. CCT137690 is a pan5 Aurora kinase inhibitor, and Bosutinib is an SRC kinase inhibitor. Within $72 \mathrm{~h}$ of administration, CCT137690 $6 \quad(200 \mathrm{nM})$ and Bosutinib (400 nM) inhibited Lepto1 cell viability by $~ 95 \%$ compared to $0.1 \%$ DMSO-treated 7 controls.

8 C.) Dose-dependent inhibition of Lepto1 cell viability by CCT137690 (left) and Bosutinib (right), measured via

D.) Immunofluorescence images of Annexin V staining (green) in Lepto1 cells after treatment with CCT137690 $(100 \mathrm{nM})$ or $0.1 \%$ DMSO (CTL). Scale bar=50 $\mu \mathrm{m}$.

E.) Schematic showing crosstalk between Aurora-A kinase (AURKA) and SRC kinase and GM-CSF signaling to regulate downstream effectors that function in Lepto1 cell proliferation.

F.) RT-qPCR analyses of Aurora-A levels in nodular HER2+ LC tissues, primary tumor tissues (PT2), metastatic tumor tissues (MT2) or normal breast and brain tissues. Aurora-A levels are markedly elevated in nodular HER2+ LC tissues.

G.) Western blot analysis of the indicated signaling factors in Lepto1 cells treated for $48 \mathrm{~h}$ with anti-GM-CSF neutralizing antibodies (50 ng/mL) plus CCT137690 (100nM; left panel) or Bosutinib (100 nM; right panel). Control samples were left untreated, and tubulin served as a loading control.

H.) Schematic showing the protocol used for the implantation of mCherry:LUC-labeled Lepto1 cells (100K) into the cisternae magna of NOD/SCID mice, followed by the intrathecal administration of anti-GM-CSF neutralizing antibodies, with or without CCT137690 (100 mg/kg), on Days 5, 10, and 15. Tumor growth was monitored by BLI from Days 15 to 50 .

I.) (Left panel) BLI analysis of mCherry:LUC-labeled Lepto1 tumors in mice treated with anti-GM-CSF antibody alone or with CCT137690. Control animals were treated with vehicle (Diluent PBS or 0.1\% DMSO). (Right panel) Survival analysis of mice used for BLI analysis $(n=9)$. Combination treatment with anti-GM-CSF antibodies and CCT137690 significantly significant reduced tumor volume and increased survival. Treatment with anti-GM-CSF antibodies alone also reduced tumor growth significantly but to a lesser extent. ${ }^{* *} \mathrm{p}<$ 0.01 . 


\section{Figures}

A

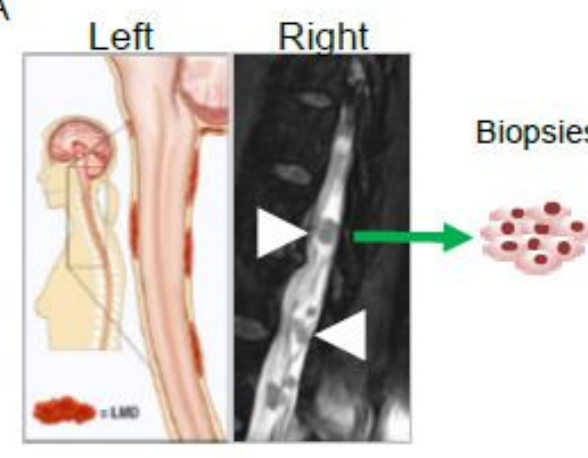

B

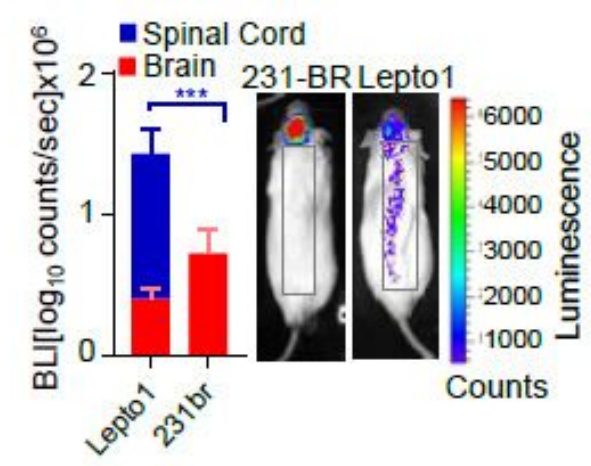

C

E

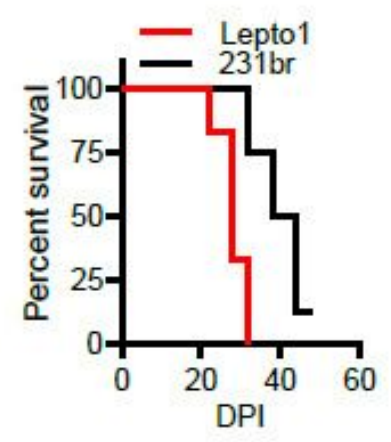

D Pre implanted Lepto1
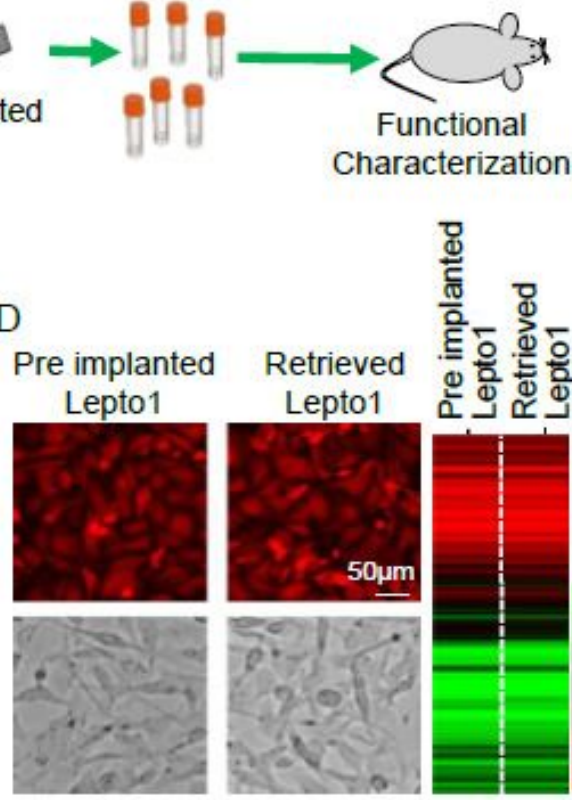

Characterization

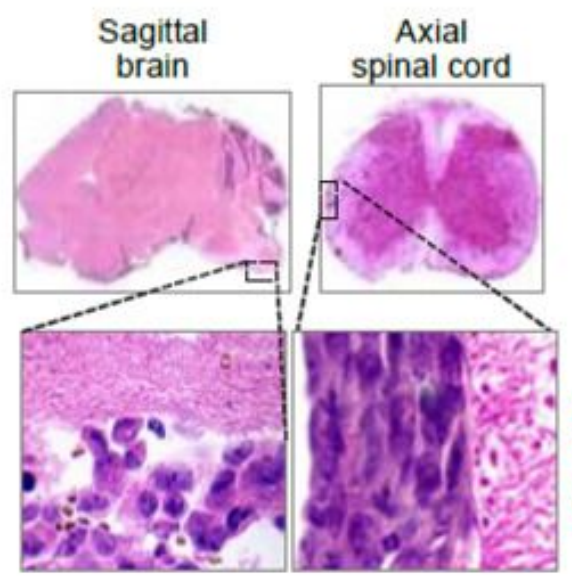

F

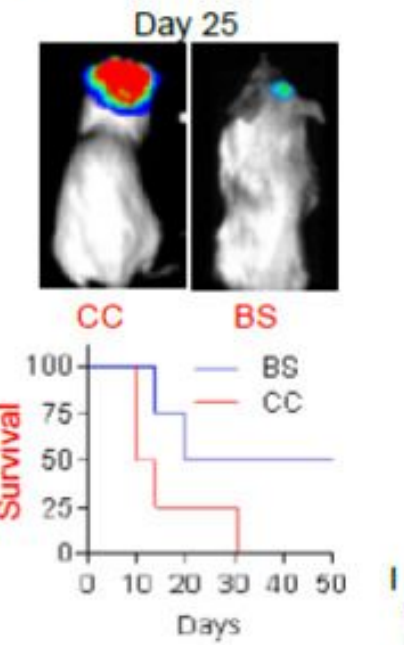

G

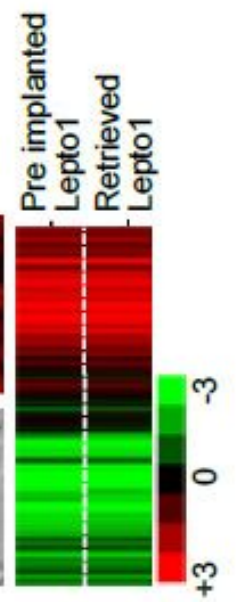

$\mathrm{H}$
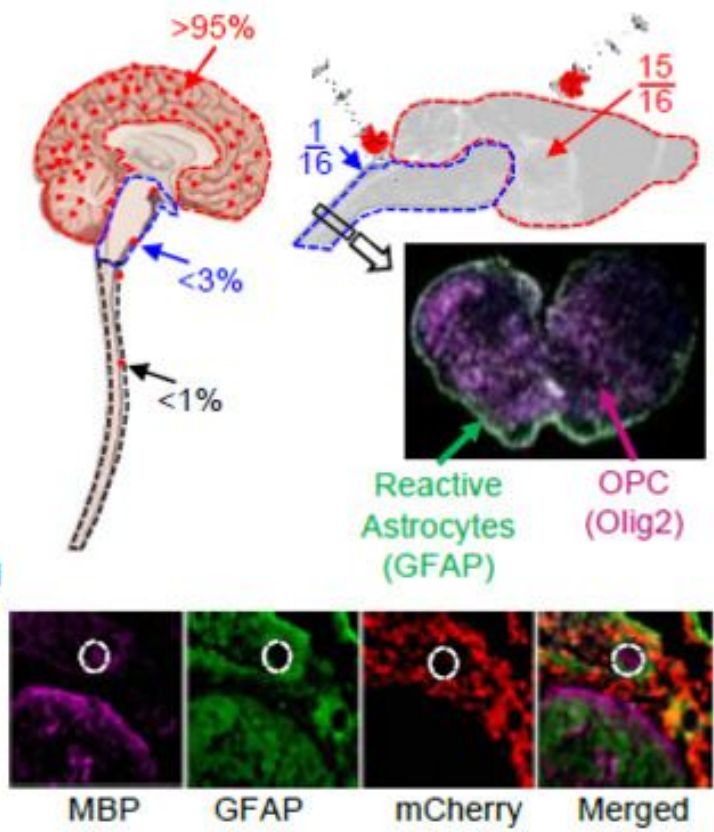

Figure 1

Derivation and characterization of nodular HER2+ LC cells. See PDF for figure legend. 


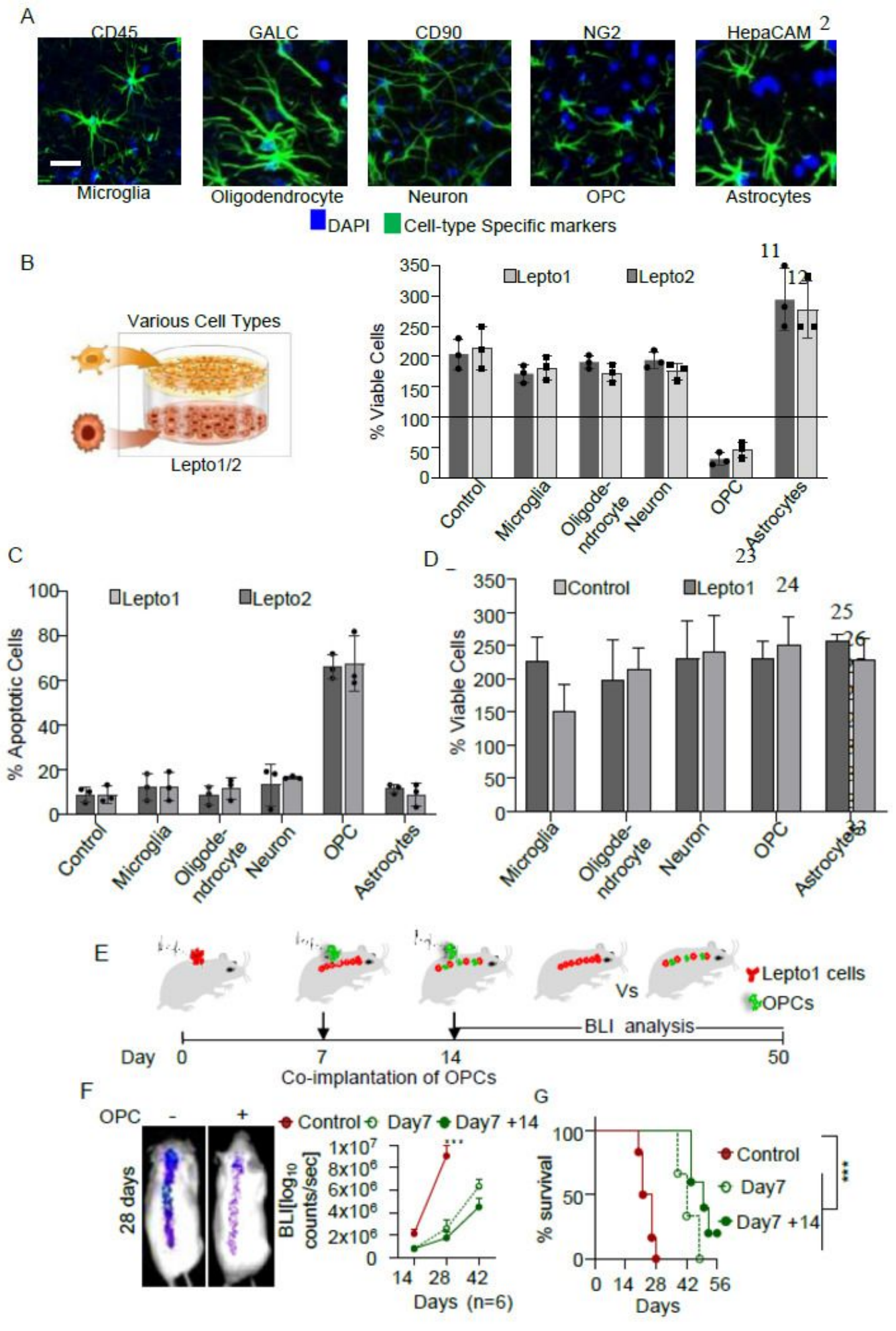

Figure 2

The presence of OPCs reduces HER2+ LC cell viability. See PDF for figure legend. 

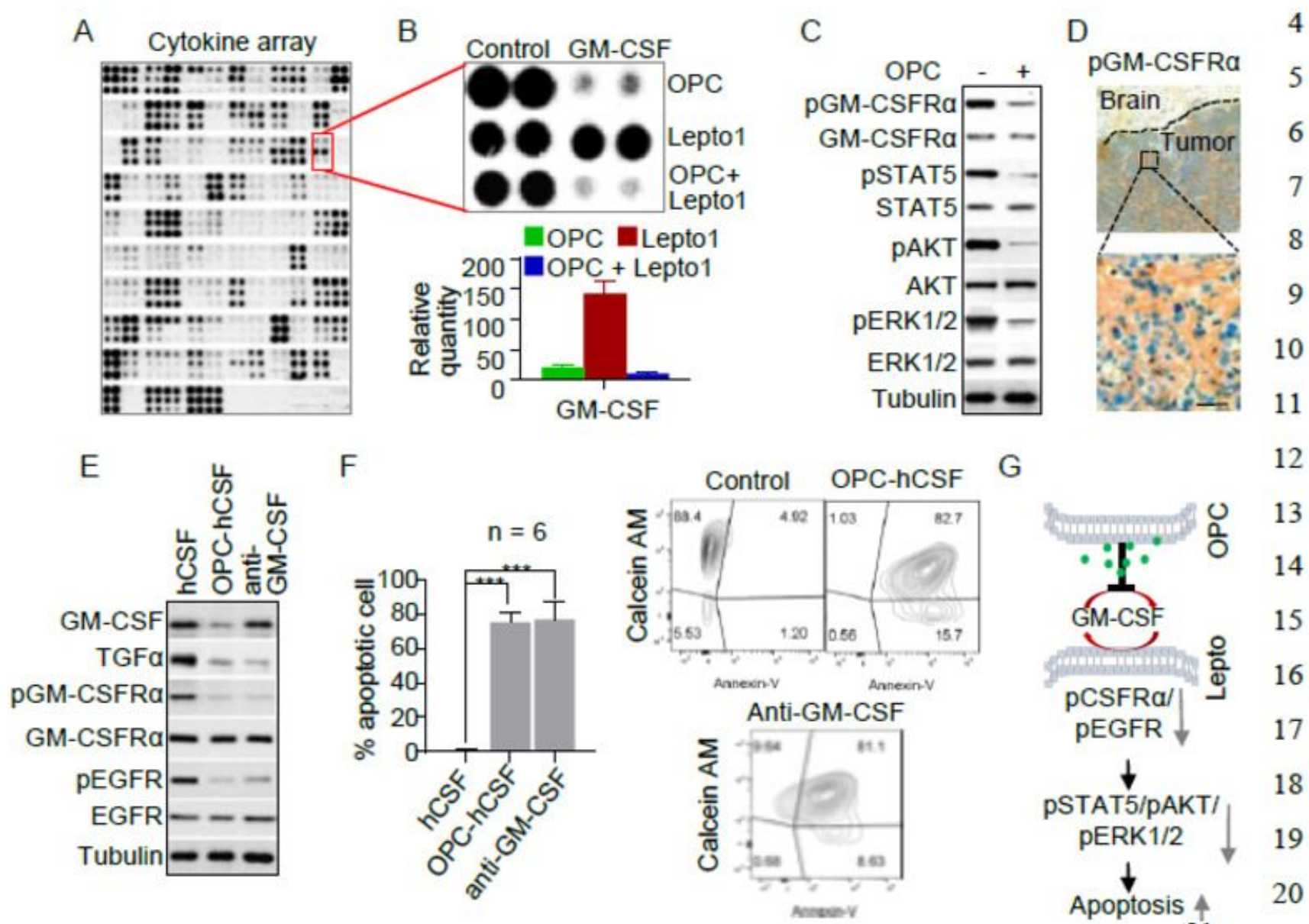

G

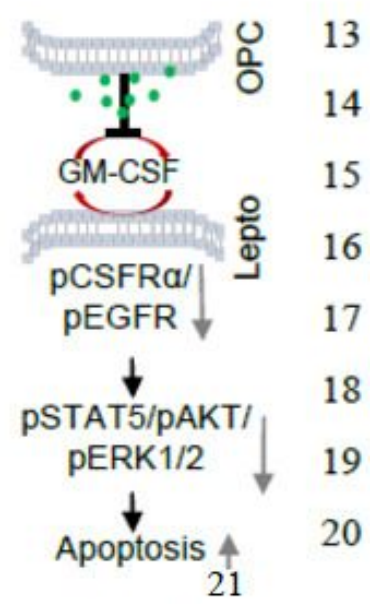

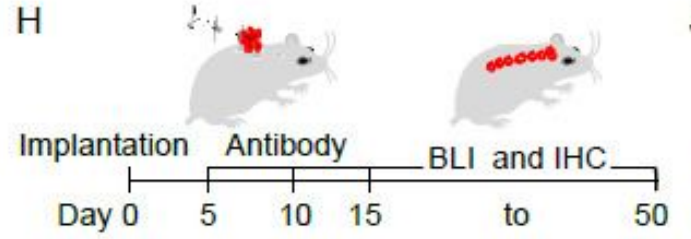
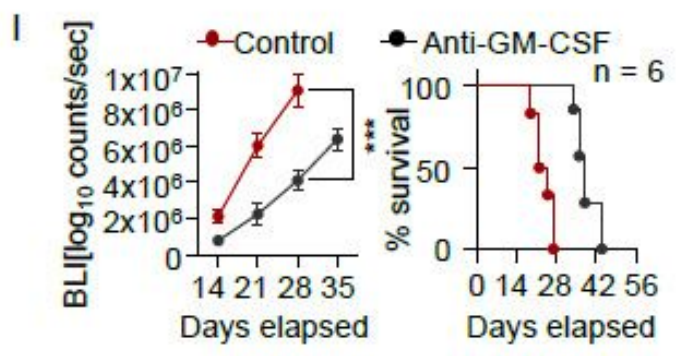

J
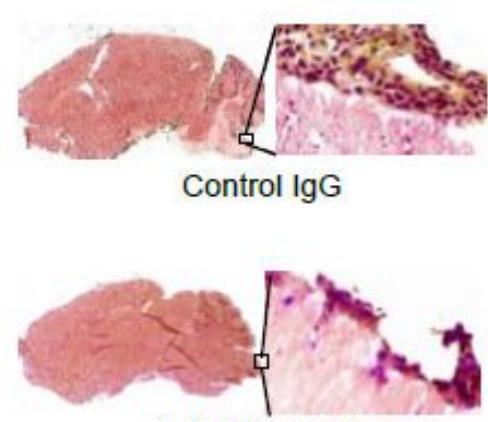

Anti-GM-CSF IgG
$\mathrm{K}$

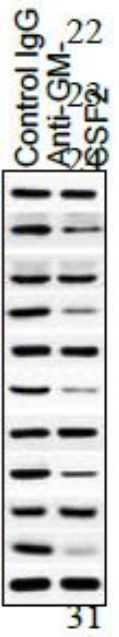

\section{Figure 3}

GM-CSF acts as an oncogenic autocrine driver of HER2+ LC cell growth. See PDF for figure legend. 
A
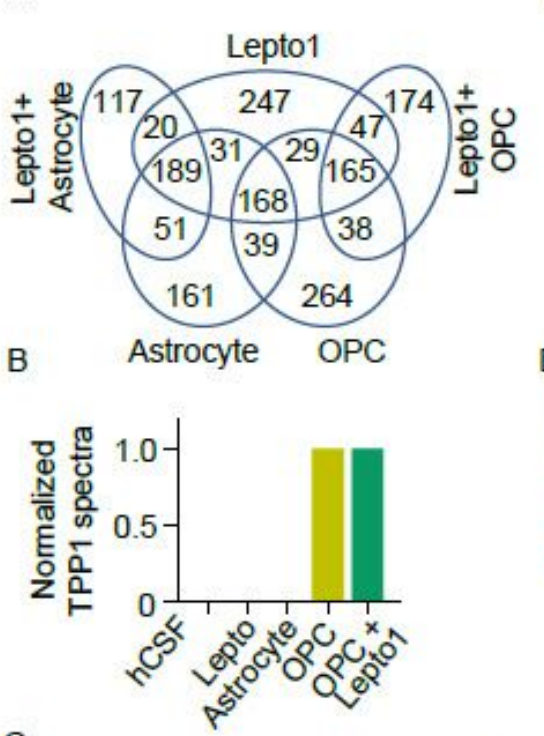

G

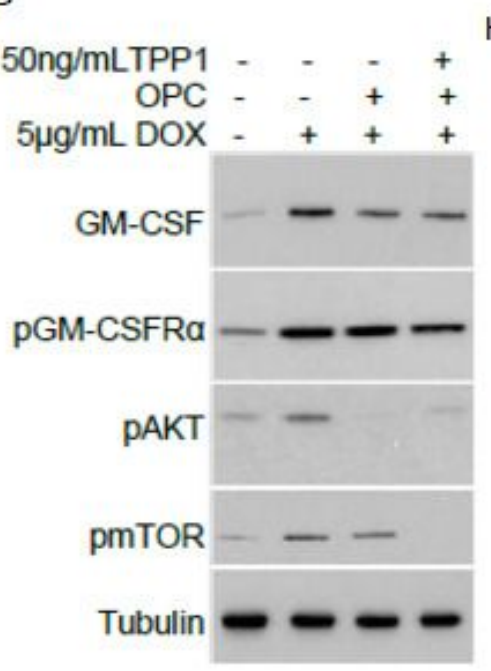

C
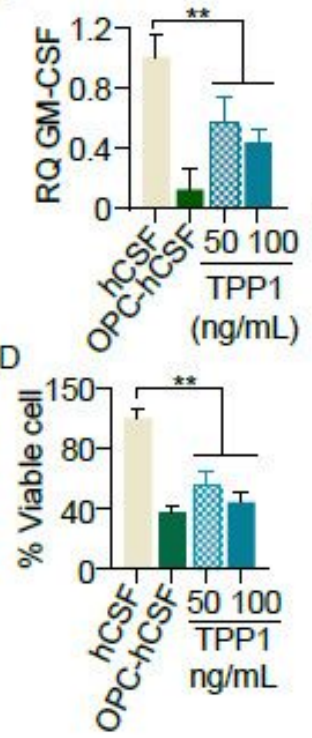

$\mathrm{H}$

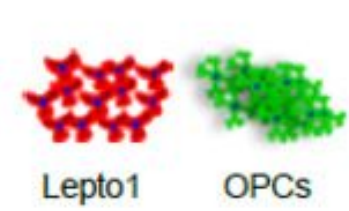

E

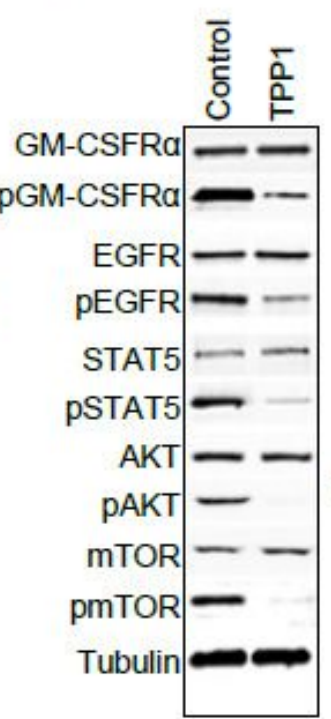

F 5

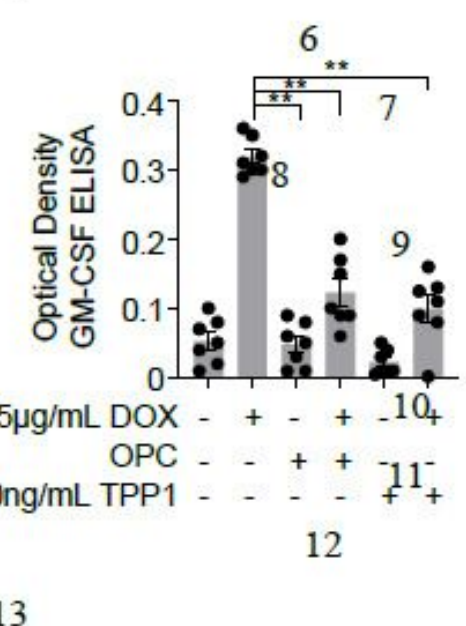

15

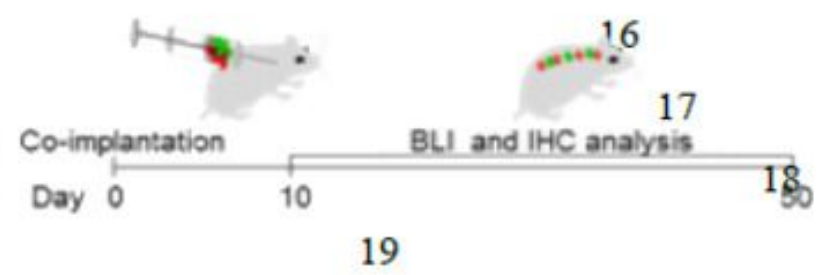

I 20
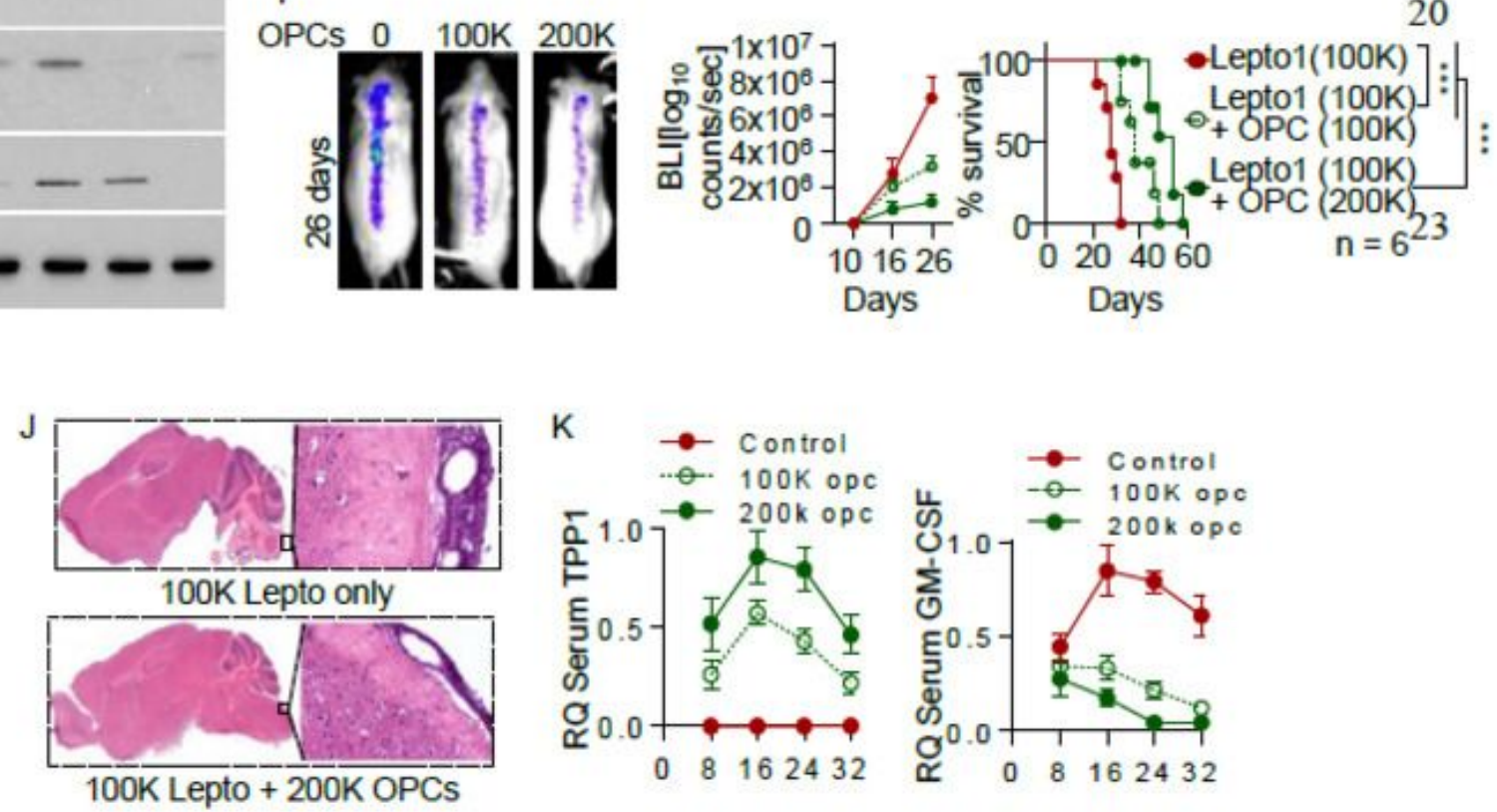

Figure 4

OPC-derived TPP1 is a candidate regulator of GM-CSF in HER2+ LC cells. See PDF for figure legend. 
A

384 well plate

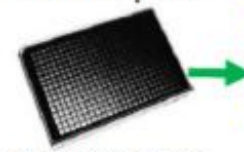

Seeding; Dayo

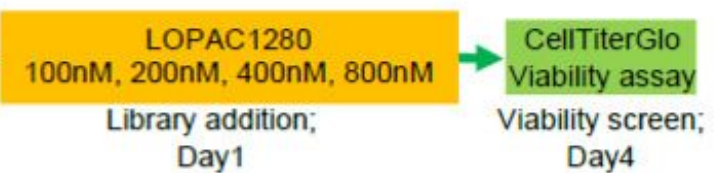

Day1

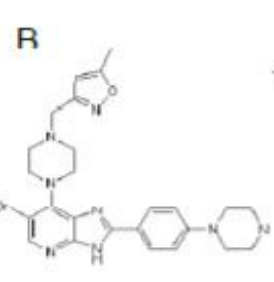

СCT137690
3

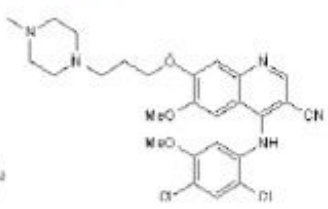

Bosutinib

5

C
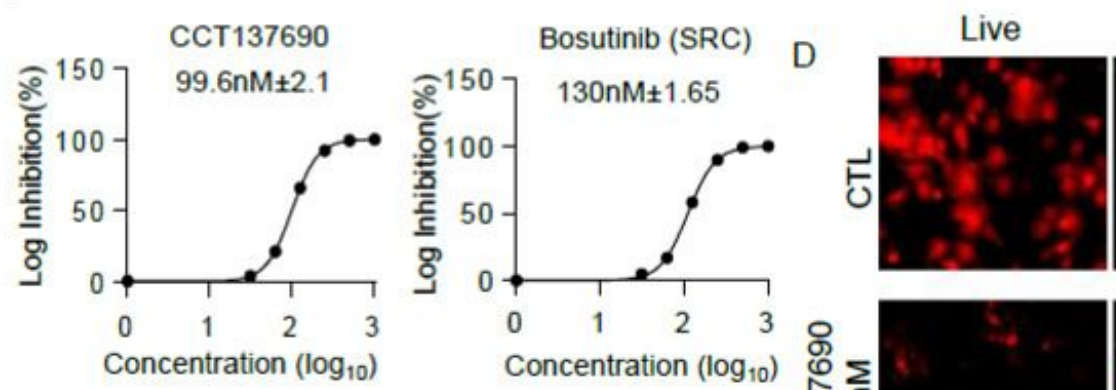

Apoptotic

DAPI

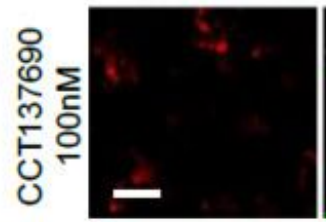

E

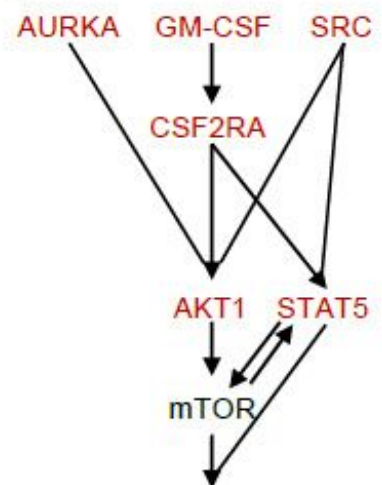

Downstream targets

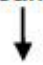

Cell proliferatipn

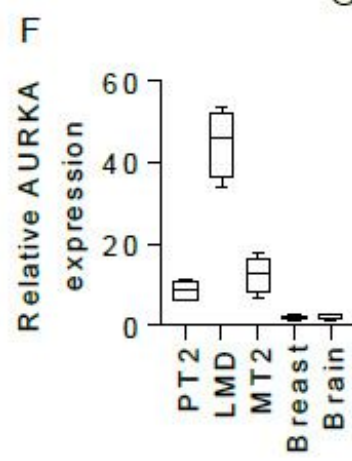

G

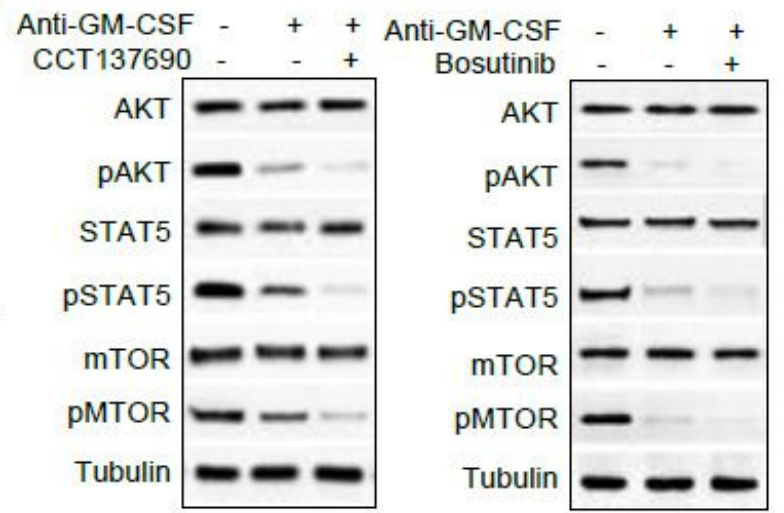

$\mathrm{H}$

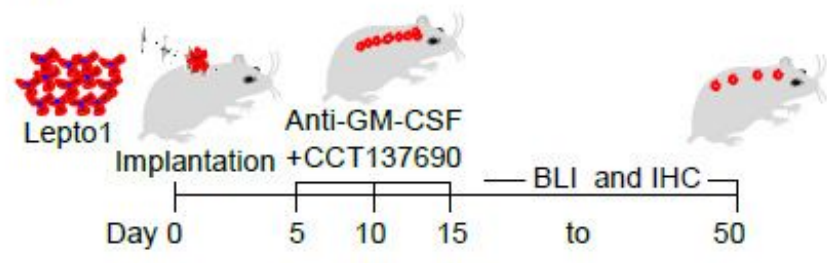

1

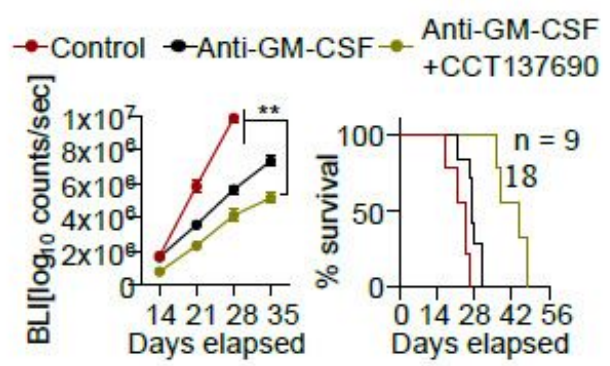

\section{Figure 5}

Combination treatment with a pan-Aurora kinase inhibitor and anti-GM-CSF neutralizing 1 antibodies antagonizes Lepto cell growth in vivo. See PDF for figure legend.

\section{Supplementary Files}


This is a list of supplementary files associated with this preprint. Click to download.

- AnsarietalSupplementaryNComm.pdf 